\title{
Dominant-negative IKZF1 mutations cause a T, B, and myeloid cell combined immunodeficiency
}

David Boutboul, ${ }^{1}$ Hye Sun Kuehn, ${ }^{2}$ Zoé Van de Wyngaert,, ${ }^{1,3}$ Julie E. Niemela, ${ }^{2}$ Isabelle Callebaut, ${ }^{4}$ Jennifer Stoddard, ${ }^{2}$ Christelle Lenoir, ${ }^{1}$ Vincent Barlogis, ${ }^{5}$ Catherine Farnarier, ${ }^{6}$ Frédéric Vely, ${ }^{7}$ Nao Yoshida, ${ }^{8}$ Seiji Kojima, ${ }^{9}$ Hirokazu Kanegane, ${ }^{10}$ Akihiro Hoshino, ${ }^{10}$ Fabian Hauck,, ${ }^{11}$ Ludovic Lhermitte, ${ }^{12}$ Vahid Asnafi, ${ }^{12}$ Philip Roehrs, ${ }^{13}$ Shaoying Chen, ${ }^{14}$ James W. Verbsky, ${ }^{14}$ Katherine R. Calvo, ${ }^{15}$ Ammar Husami, ${ }^{16}$ Kejian Zhang, ${ }^{16}$ Joseph Roberts, ${ }^{17}$ David Amrol, ${ }^{18}$ John Sleaseman, ${ }^{17}$ Amy P. Hsu, ${ }^{19}$ Steven M. Holland, ${ }^{19}$ Rebecca Marsh, ${ }^{16}$ Alain Fischer, ${ }^{3,20,21}$ Thomas A. Fleisher, ${ }^{2}$ Capucine Picard, ${ }^{1,22}$ Sylvain Latour, ${ }^{1}$ and Sergio D. Rosenzweig ${ }^{2}$

'Laboratory of Lymphocyte Activation and Susceptibility to EBV Infection, Inserm UMR 1163, Paris, France. ${ }^{2}$ Immunology Service, Department of Laboratory Medicine, Clinical Center, NIH, Bethesda, Maryland, USA. ${ }^{3}$ University Paris Descartes Sorbonne Paris Cité, Imagine Institute, Paris, France. ${ }^{4}$ Centre National de la Recherche Scientifique UMR 7590 , Sorbonne Universities, University Pierre et Marie Curie-Paris 6-MNHN-IRD-IUC, Paris, France. ${ }^{5}$ Department of Paediatric Haematology-Oncology, La Timone Hospital, Marseille, France. ${ }^{6}$ Assistance Publique - Hôpitaux de Marseille (APHM) Hôpital Timone Enfants, Service d'Immunologie - Marseille Immunopôle, Marseille, France. ${ }^{7}$ Aix Marseille University, APHM, CNRS, Inserm, Centre d'Immunologie de Marseille-Luminy (CIML), Hôpital Timone Enfants, Service d'Immunologie - Marseille Immunopôle, Marseille, France. ${ }^{8}$ Department of Hematology and Oncology, Children's Medical Center, Japanese Red Cross Nagoya First Hospital, Nagoya, Japan. ${ }^{9}$ Department of Pediatrics, Nagoya University Graduate School of Medicine, Nagoya, Japan. ${ }^{10}$ Department of Pediatrics and Developmental Biology, Graduate School of Medical and Dental Sciences, Tokyo Medical and Dental University, Tokyo, Japan. "Department of Pediatric Immunology and Rheumatology, Dr. von Hauner Children's Hospital, Ludwig-Maximilians-Universität (LMU), Munich, Germany. ${ }^{12}$ University Paris Descartes Sorbonne Cité, Institut Necker-Enfants Malades (INEM), Inserm 1151 and Laboratory of Onco-Hematology, Assistance Publique-Hôpitaux de Paris (APHP), Necker-Enfants Malades Hospital, Paris, France. ${ }^{13}$ Levine Children's Hospital, Carolinas Healthcare System, Charlotte, North Carolina, USA. ${ }^{14}$ Department of Pediatrics, Division of Rheumatology, Medical College of Wisconsin, Madison, Wisconsin, USA. ${ }^{15}$ Hematology section, Department of Laboratory Medicine, Clinical Center, NIH, Bethesda, Maryland, USA. ${ }^{16}$ Division of Human Genetics and Division of Immune Deficiency and Bone Marrow Transplant, Cincinnati Children's Hospital, Cincinnati, Ohio, USA. ${ }^{17}$ Department of Pediatrics, Duke University Medical Center, Durham, North Carolina, USA. ${ }^{18}$ University of South Carolina School of Medicine, Columbia, South Carolina, USA. ${ }^{19}$ Laboratory of Clinical Infectious Diseases, NIAID, NIH, Bethesda, Maryland, USA. ${ }^{20}$ Department of Pediatric Immunology, Hematology and Rheumatology, Necker-Enfants Malades Hospital, APHP, Paris, France. ${ }^{21}$ Collège de France, Paris, France. ${ }^{22}$ Centre d'Etude des Déficits Immunitaires, Necker-Enfants Malades Hospital, APHP, Paris, France.

Ikaros/IKZF1 is an essential transcription factor expressed throughout hematopoiesis. IKZF1 is implicated in lymphocyte and myeloid differentiation and negative regulation of cell proliferation. In humans, somatic mutations in IKZF1 have been linked to the development of B cell acute lymphoblastic leukemia (ALL) in children and adults. Recently, heterozygous germline IKZF1 mutations have been identified in patients with a B cell immune deficiency mimicking common variable immunodeficiency. These mutations demonstrated incomplete penetrance and led to haploinsufficiency. Herein, we report 7 unrelated patients with a novel early-onset combined immunodeficiency associated with de novo germline IKZF1 heterozygous mutations affecting amino acid N159 located in the DNA-binding domain of IKZF1. Different bacterial and viral infections were diagnosed, but Pneumocystis jirovecii pneumonia was reported in all patients. One patient developed a T cell ALL. This immunodeficiency was characterized by innate and adaptive immune defects, including low numbers of B cells, neutrophils, eosinophils, and myeloid dendritic cells, as well as T cell and monocyte dysfunctions. Notably, most T cells exhibited a naive phenotype and were unable to evolve into effector memory cells. Functional studies indicated these mutations act as dominant negative. This defect expands the clinical spectrum of human IKZF1-associated diseases from somatic to germline, from haploinsufficient to dominant negative.

\section{Introduction}

IKAROS, a transcription factor encoded by IKZF1, is expressed during hematopoiesis $(1,2)$. It has been involved in positive regulation of lymphocyte differentiation and negative regulation of cell proliferation (3). IKAROS (designated herein as IKZF1) also plays some role in peripheral lymphocyte homeostasis, mainly by favoring a Th2 response in T cells and negatively

Authorship note: DB and HSK, as well as SL and SDR, contributed equally to this work. Conflict of interest: The authors have declared that no conflict of interest exist. Submitted: October 19, 2017; Accepted: April 17, 2018.

Reference information: J Clin Invest. 2018;128(7):3071-3087.

https://doi.org/10.1172/JCI98164 regulating follicular B cell activation $(4,5)$. Additional roles of IKZF1 in the development of myeloid cells, including neutrophils, basophils, platelets, and red blood cells, have been shown in different models (6-9).

IKZF1 is mainly composed of 2 functional domains: an $\mathrm{N}$-terminal DNA-binding domain (DBD) formed by 4 zinc fingers $(\mathrm{ZF})$ and a C-terminal dimerization domain consisting of 2 additional ZFs. More than 10 isoforms of IKZF1 have been described and can be found in normal hematopoietic cells. Some isoforms lack one or more DBD ZF and are considered to behave as dominant negative (10). IKZF1 binding to DNA occurs at pericentromeric heterochromatin (PC-HC) regions; IKZF1 functions primarily as a gene repressor by interacting with the 
NurD complex $(11,12)$. IKZF1 acts as a homodimer, but it may also work as a heterodimer by associating with other IKAROS isoforms or family members, such as IKZF2 (HELIOS), IKZF3 (AIOLOS), and IKZF4 (EOS) (3).

Several mouse models have been generated in the past 20 years and confirmed the key role of IKZF1 in lymphoid and myeloid development. Ikzf1-deficient null mice in which the dimerization domain was removed showed a severe lymphoid development arrest (13). Ikzf1-deficient null mice also exhibited myeloid development abnormalities, including absence of DCs, increased basophils, and increased megakaryocytes $(7,8,13)$. Ikzf1 ${ }^{\mathrm{L} / \mathrm{L}}$ mice, expressing low levels of Ikaros, showed increased myeloid precursors with impaired migratory potential and increased susceptibility to cell death at the embryonic stage; adult mice showed a near 2-fold reduction in peripheral blood neutrophils (6). ENU-induced Plastic mice harboring the missense mutation p.H191R in the DBD ZF3 in homozygosity showed embryonic lethality with severe anemia and defects in granulocyte differentiation, increased macrophage formation, and blocked lymphoid development. Heterozygous animals had normal lymphoid development until the second month of life and then invariably developed T cell lymphoid malignancy, which underlines the role of murine Ikaros in controlling lymphoid proliferation $(10,13-15)$. The severity of this dominant-negative effect at the heterozygous state was linked to its action over the WT Ikaros allele and also toward Aiolos (14).

In humans, somatic IKZF1 mutation mainly by deletion has been linked to B cell ALL (B-ALL) development in children and adults and constitutes an adverse prognostic factor in Philadelphia chromosomepositive pediatric B-ALL $(16,17)$. More recently, germline mutations have been described in patients with common variable immunodeficiency (CVID) associated with B cell immune deficiency, B-ALL susceptibility, and autoimmune manifestations $(18,19)$. Although no clinical $\mathrm{T}$ cell defects were evident among these patients, elevated naive and central memory $\mathrm{CD}^{+} \mathrm{CD}^{+} \mathrm{T}$ cells not related to increased cellular proliferation, decreased cell death, clonal expansion, or specific viral infections were detected. All mutations were heterozygous with incomplete penetrance and included deletions and missense mutations affecting IKZF1 DBD. Functional studies showed that these mutations acted by haploinsufficiency (18).

In the present study, we describe a new early-onset combined immunodeficiency (CID) syndrome caused by particular de novo heterozygous germline IKZF1 mutations detected in 7 unrelated patients. Myeloid defects were also a prominent part of the biological picture. All the mutations were located in ZF2, affecting the IKZF1 DBD, and in vitro functional studies demonstrated these to be dominant-negative mutations.

\section{Results}

Identification of heterozygous IKZF $1^{\text {N159S/T }}$ mutations in 7 patients with combined immunodeficiency. Whole-exome sequencing was performed in patients with genetically uncharacterized CID from France, Japan, and the United States. Seven patients carried heterozygous IKZF1 missense mutations at position chr7:50450292: 6 presented with a c. $476 \mathrm{~A}>\mathrm{G}$ transition leading to an asparagineto-serine change at amino acid 159 (p.N159S) and 1 an A $>C$ transversion leading to an asparagine-to-threonine change at the same site (p.N159T) (Figure 1, A and B). IKZF1 N159S or T mutations were not found in public exome databases. Mutations were confirmed by Sanger sequencing and analyzed in the available family members. No such changes were detected in the relatives tested, which suggests the mutations were de novo in at least 6 of the 7 patients (Figure 1A). Of note, the 2 Japanese patients (families $\mathrm{B}$ and $\mathrm{F}$ ) have been previously reported, but the relationship between their IKZF1 genotype and their clinical phenotypes was not to our knowledge examined in depth $(2,19)$.

Amino acid sequence alignment of the $4 \mathrm{ZF}$ domains contained in the DBD of IKZF1 indicated that the residue N159 and the other residues previously involved in haploinsufficient mutations are at positions $-1,-4,-7$, and -8 relative to the first histidine in the $4 \mathrm{ZF}$ domains (Figure 1C). All these positions are known to be involved in DNA binding (20) and are highly conserved across species, which highlights their importance for the function of IKZF1 (Figure 1D). Supporting a key role of the N159 residue, p.N159A mutation was previously tested and shown to behave as a dominant-negative mutation in vitro, while its effect in vivo has not been described in animal models or extensively evaluated in humans $(18,21)$.

Clinical and immunologic phenotype of patients with heterozygous IKZF1 $1^{\text {N159S/T }}$ mutations. All patients carrying IKZF1 N159 heterozygous missense mutations became symptomatic early in life: 3 within their first 6 months of life, and all of them by the age of 15 months. Pneumocystis jirovecii pneumonia was diagnosed in all patients between the ages of 6 and 24 months; this was the first clinical manifestation in 2 and occurred multiple times in 2 patients (Table 1). Other infectious complications included invasive bacterial respiratory tract infections in 6 patients, severe or recurrent viral infections in 5 , superficial or invasive fungal infections in 4, and liver cryptosporidiosis associated with sclerosing cholangitis and secondary cirrhosis in 1 patient (A1). No autoimmune diseases were identified. T cell acute lymphoblastic leukemia (T-ALL) was diagnosed in 1 patient (F1) at the age of 13 years (2). Three patients received hematopoietic stem cell transplants (HSCTs) before their genetic diagnosis was established, 2 for CID during childhood and the other for T-ALL (2). Despite transplantation, one of the patients with severe immune deficiency died from infectious complications (R. Marsh, unpublished observations).

Profound hypogammaglobulinemia affecting the major isotypes and severe B cell lymphopenia were detected in 7 patients at diagnosis of immunodeficiency (Table 2). IgE was low or undetectable in 5 of them. Severe $\mathrm{T}$ cell lymphopenia was found in 2 (E1 and transiently during early infancy in F1, although it was originally diagnosed as severe combined immunodeficiency; ref. 2), and $\mathrm{T}$ cell lymphocytosis affecting both $\mathrm{CD}^{+} \mathrm{CD}^{+}$and $\mathrm{CD}^{+} \mathrm{CD}^{+}$cells was detected in 3 patients. Most of the circulating T cells had a naive phenotype $\left(\mathrm{CD} 45 \mathrm{RA}^{+} \mathrm{CD} 62 \mathrm{~L}^{+}\right.$or $\mathrm{CD} 45 \mathrm{RA}^{+}$ $\mathrm{CD}^{2} \mathrm{RO}^{-}$) (Figure 2A, Table 2, and Supplemental Figure 1; supplemental material available online with this article; https://doi. org/10.1172/JCI98164DS1), although CD45RA was expressed at low density (Figure 2, A and B, and Supplemental Figure 1). These cells also exhibited the phenotype of recent thymic emigrants $\left(\mathrm{CD}_{4} 5 \mathrm{RA}^{+} \mathrm{CD} 31^{+}\right)$(Table 2 and Figure $2 \mathrm{C}$ ), with the exception of cells from patient E1 (the only adult in our series who also was found to have a hypocellular bone marrow) (Table 2). Inversely, central memory and effector memory $\mathrm{T}$ cells were decreased in 
A

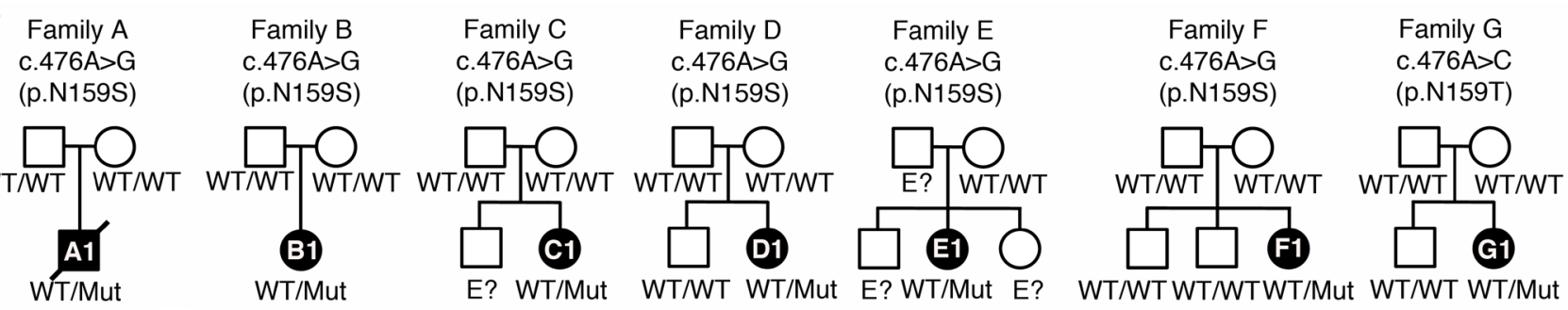

B

Families A-F

c. $476 A>$ G, p.N159S

Family $\mathrm{G}$

CADD score $=24$

c. $476 \mathrm{~A}>\mathrm{C}, \mathrm{p} . \mathrm{N} 159 \mathrm{~T}$

CADD score $=26.7$

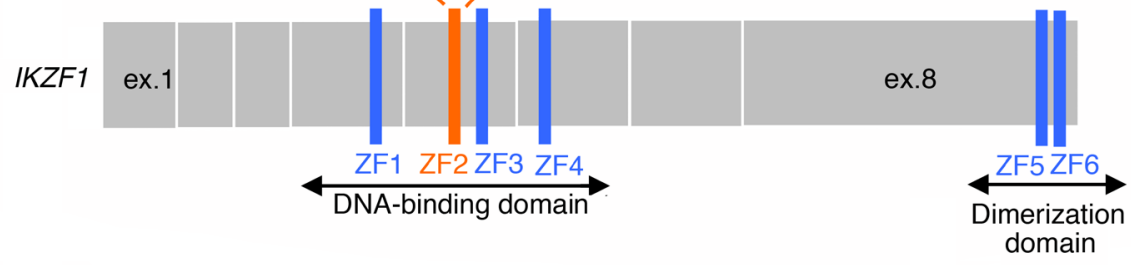

C

$\overline{8} \overline{7} \quad \overline{5} \overline{4} \quad \overline{1}$

ZF1 : NG KL KCD ICGI I C I G P NVIMVHKR S . HT G

ZF2 : ERPF Q CNQCGASFTQKGNLLRHIKL . HS G

ZF3: E K P F K CH L CNY A CR R D ALT GHLRT . HS V

ZF4：G K P H KCG Y CGR S Y K QR S S LE EHK E R CHNY

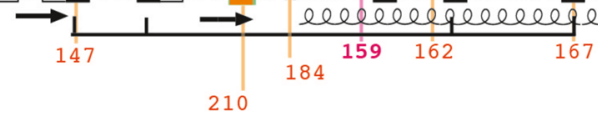

D

IKZF 1 HUMAN

IKZF 1 MOUSE

IKZF 1 CHICKEN

IKZF1 ONCMY

IKZF1 DANRE

IKZF2 HUMAN

IKZF2 MOUSE

IKZF2 SKATE

IKZF 3 HUMAN

IKZF 3 MOUSE

IKZF 3 BOVIN

IKZF3 SKATE

IKZF4 HUMAN

IKZF4 MOUSE

IKZF HAGFISH

IKZF OIKOPLEURA

IKZF CIONA

IKZF LAMPREY

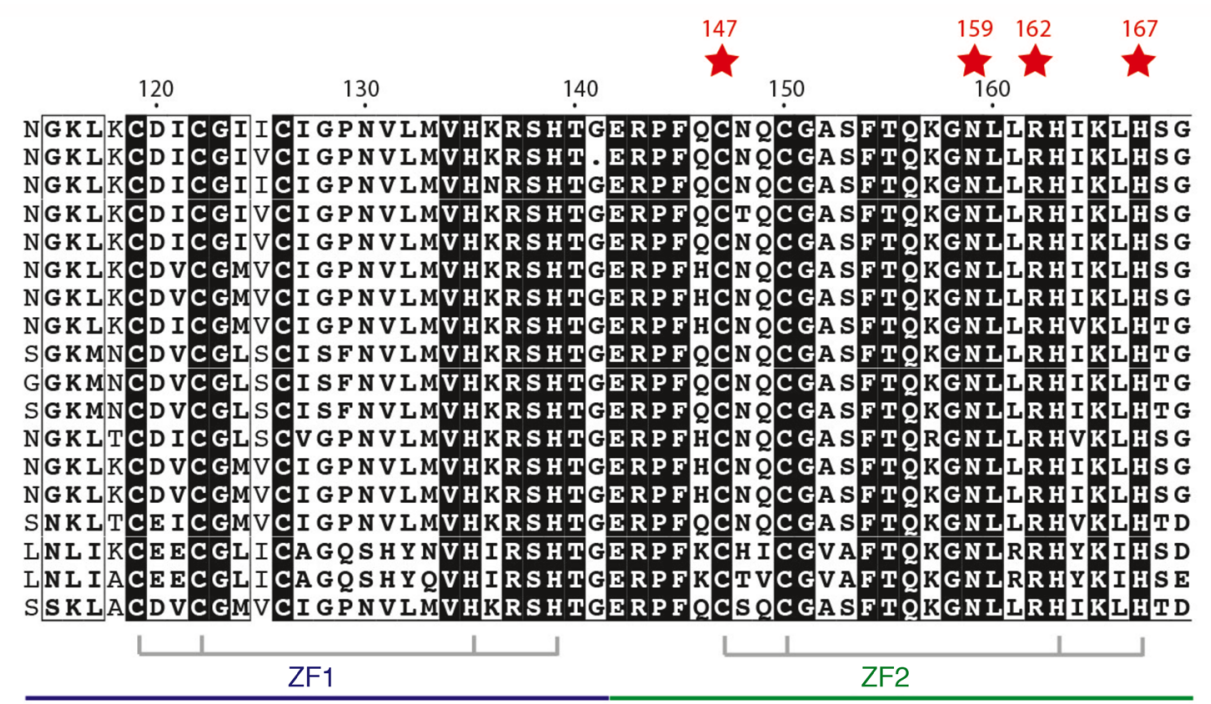

Figure 1. Pedigree analysis in patients with IKZF1 ${ }^{N 1595 / T}$ mutations. (A) Pedigrees of 7 kindreds with $/ K Z F 7^{N 1595 / T}$ mutations (families A-G). Affected individuals are shown in black. Diagonal lines indicate deceased individuals. WT, wild-type allele; Mut, mutant allele. "E?" indicates unknown genotype. (B) Schematic representation of the IKZF1 coding region, from exon 1 to 8 , and corresponding domains, shown in dark gray: the DNA-binding domain containing 4 ZFs and the dimerization domain containing 2 ZFs. Sites of mutations are shown on top with predicted combined annotation dependent depletion (CADD) scores. (C) Alignment of the ZF1-4 DNA-binding domains of IKZF1. Two cysteines and 2 histidines (C2H2) in each finger are responsible for $Z^{2+}$ coordination (bottom connecting lines), except for ZF4, in which the last histidine is replaced by a cysteine (strictly conserved in the Ikaros-like family). Amino acids at positions $-1,-4,-7$, and -8 relative to the first histidine (i.e., 0 ) are known to be involved in DNA binding (19). Mutated amino acids are colored in pink (this study) or orange (previous studies). The ZF predicted secondary structures (deduced from the model of $3 D$ structure shown in Supplemental Figure 8) are shown below the alignment. (D) Alignment of ZF domains of IKZF1 family members including IKZF1/ IKAROS, IKZF2/HELIOS, IKZF3/AIOLOS, and IKZF4/EOS. Sequences of species from the Chordata phylum including marine Urochordata (Ciona and Oikopleura) and primitive fish such as hagfish are aligned. Red stars indicate residues found to be mutated in patients. All these residues are highly conserved through species. 


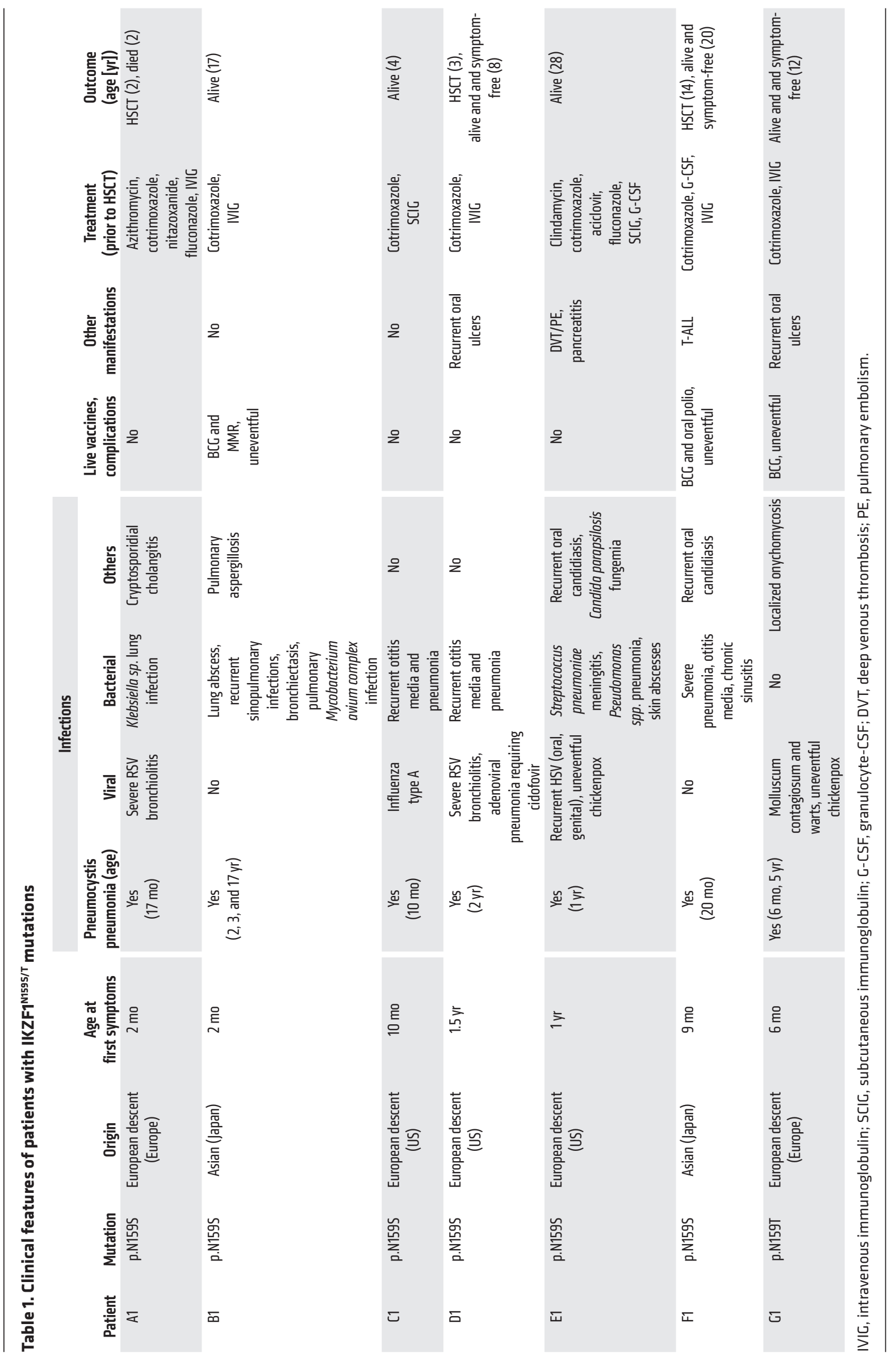


the patients tested (Table 2 and Figure 2A). Double-negative T (DNT) cells were elevated in the 2 patients tested. T cell receptor $\mathrm{V} \beta$ (TCR-V $\beta$ ) flow cytometric analysis was performed in 4 patients, and TCR-V $\alpha$ spectratyping analysis was done in 1 (data not shown). Neither of these tests revealed any abnormalities, which suggests that the $\mathrm{T}$ cell repertoire is normal and unaffected in patients. NK cell count was normal or slightly reduced in all patients. Eosinophils were undetectable in 6 patients tested. Mild to moderate chronic neutropenia was detected in 5 of 7 patients as early as in their second year of life, and myeloid DCs were reduced in the 2 patients tested (Table 2 and see below).

$T$ cell defects in patients with heterozygous IKZF1 $1^{\text {N159S/T }}$ mutations. The in vitro immunophenotype of $\mathrm{T}$ cells from patients and healthy controls was evaluated by flow cytometry on peripheral blood mononuclear cells (PBMCs) that were phytohemagglutinin-stimulated (PHA-stimulated) and maintained in culture for 10 days (Figure 2D). Naive T cells from healthy controls progressively declined to less than $2 \%$ at the end of the study, but naive $\mathrm{T}$ cells from patients remained consistently high at more than $38 \%$ during the entire culture period. Inversely, effector memory $\mathrm{T}$ cells progressively increased at least to $44 \%$ in healthy controls, as patients' cells remained low at less than $10 \%$ after 10 days in culture. While central memory $\mathrm{T}$ cells were decreased at time 0 in patient cell cultures, they reached levels similar to those of healthy controls at the end of the study. A differential analysis of targeted RNA sequencing (RNA-Seq) data was evaluated between unstimulated naive $\mathrm{CD} 4^{+} \mathrm{T}$ cells from 2 patients carrying IKZF1 $1^{\mathrm{N} 159 \mathrm{~S} / \mathrm{T}}$ mutants (C1 and G1), 5 healthy controls, and a patient carrying haploinsufficient mutation IKZF1 $1^{\mathrm{H} 167 \mathrm{R}}$. Correlation analysis, hierarchical clustering, and principal component analysis revealed significantly differentially expressed transcripts between cells carrying mutants IKZF1 ${ }^{\mathrm{N} 159 \mathrm{~s} / \mathrm{T}}$ when compared with either healthy control or $\mathrm{IKZF} 1^{\mathrm{H} 167 \mathrm{R}}$ naive $\mathrm{CD} 4^{+} \mathrm{T}$ cells. No differences were detected when healthy control and IKZF1 ${ }^{\mathrm{H} 167 \mathrm{R}}$ cells were compared with each other (Supplemental Figure 2A). Proliferation of patients' $T$ cells was markedly reduced in response to a low dose of immobilized anti-CD3 $(1 \mu \mathrm{g} / \mathrm{ml})$, soluble anti-CD3 plus CD28 $(1 \mu \mathrm{g} / \mathrm{ml}$ each), or antigen stimulation with tetanus toxoid or candida antigen (Figure 3A, Figure 4C, and Table 2). In contrast, $\mathrm{T}$ cell proliferation to $\mathrm{PHA}$, a high dose of immobilized anti-CD3 $(10 \mu \mathrm{g} / \mathrm{ml})$, or anti-CD3/CD28-coated beads was normal. Addition of IL-2 did not rescue the proliferation defect (Figure 3B). While the expression of IL-2 receptor chains CD25, CD122, and CD132 was readily detected on the surface of the patients' $T$ cells (Supplemental Figure 3A and data not shown), CD25 was not upregulated on $\mathrm{T}$ cells after stimulation with a low dose of immobilized anti-CD3 with or without IL-2 (Figure 3C). Moreover, STAT5 phosphorylation upon IL-2 stimulation was markedly impaired in $\mathrm{CD} 4^{+}$cells, a pattern that could also reflect the strong reduction of $\mathrm{CD} 4^{+}$memory $\mathrm{T}$ cells in the patients (Supplemental Figure 3B). These observations strongly suggest that defective CD25 upregulation and the unresponsiveness to IL-2 contribute to defective $\mathrm{T}$ cell proliferation in addition to the reduced response to low doses of anti-CD3. Consistent with an impaired response to $\mathrm{IL}-2$, natural regulatory $\mathrm{T}$ cell $\left(\mathrm{CD} 4^{+} \mathrm{CD} 25^{+} \mathrm{FoxP} 3^{+}\right)$ numbers were found to be diminished in $3 \mathrm{IKZF} 1^{\mathrm{N} 1595}$ patients and the IKZF1 ${ }^{\mathrm{N} 159 \mathrm{~T}}$ patient tested (Table 2 and Supplemental Figure
3A). Cytokine production by patient $\mathrm{T}$ cells was also selectively impaired. While IL-2 production by patient T cells was normal in response to either PMA/ionomycin or CD3/CD28-coated beads, a marked defect in IFN- $\gamma$, TNF- $\alpha$, IL-4, IL-6, and IL-17A production to the latter stimuli was observed (Figure 3, D-F, and Supplemental Figure 4). Taken together, these results demonstrate that germline IKZF1 $1^{\mathrm{N} 159 \mathrm{~S}}$ and IKZF1 ${ }^{\mathrm{N} 159 \mathrm{~T}}$ mutations are associated with an immune deficiency characterized by defects in effector memory $\mathrm{T}$ cell generation, $\mathrm{T}$ cell activation, signaling, proliferation as well as cytokine production after TCR engagement. Furthermore, defects in IFN- $\gamma /$ TNF- $\alpha$, IL-4/IL-6, and IL-17A production indicate that Th polarization from Th0 toward Th1, Th2, and Th17 subsets (respectively) is markedly impaired in the 3 patients tested. These defects likely reflect both the strong reduction of $\mathrm{CD}^{+}$ memory $\mathrm{T}$ cells in the patients and the accumulation of abnormal naive $\mathrm{T}$ cells with an impaired phenotype.

$B$ cell defects associated with heterozygous IKZF1 $1^{\text {N159S/T }}$ mutations. Analysis of the B cell compartment in patients with IKZF1 ${ }^{\mathrm{N} 159 \mathrm{~S}}$ mutation showed strikingly reduced B cells in peripheral blood (Table 2). In contrast, patient G1, who carries the unique germline mutation resulting in IKZF1 ${ }^{\mathrm{N} 159 \mathrm{~T}}$, showed normal although progressively declining $\mathrm{B}$ cell numbers with only a naive phenotype (Table 2). Bone marrow examination in patients C1 and D1 demonstrated a very early B cell development arrest with reduced pre-pro-B cells $\left(\mathrm{CD} 34^{+} \mathrm{CTdT}^{+} \mathrm{CD} 19^{+/}\right)$, pro-B cells $\left(\mathrm{CD} 34^{+} \mathrm{CD} 19^{+}\right)$ and mature $\mathrm{B}$ cells $\left(\mathrm{CD} 34^{-} \mathrm{CD} 19^{+}\right)$, as previously described in patients with IKZF1 haploinsufficiency (ref. 17 and Supplemental Figure 5). In contrast to those patients, plasma cells were virtually absent in their bone marrow biopsies, suggesting a more dramatic effect over B cell development (data not shown).

Myeloid defects associated with heterozygous IKZF1 $1^{\text {N159s/T }}$ mutations. Myeloid defects, indicative of a multilineage defect in hematopoiesis, were also detected in the patients (Table 2). Two patients (B1 and E1) had mild chronic anemia and 3 (A1, F1, and G1) exhibited low-normal erythrocyte mean corpuscular volumes without evidence of inflammation or iron deficiency. Hemoglobin electrophoresis performed in G1 was normal. Eosinophils were undetectable in all patients tested. In addition, 5 patients had chronic mild to moderate neutropenia (absolute neutrophil count [ANC] $<1,000 / \mu \mathrm{l}$ and $>200 / \mu \mathrm{l}$ ), 2 of them requiring G-CSF treatment with adequate responses. Bone marrow aspirates performed in patients $\mathrm{C} 1$ and $\mathrm{D} 1$ because of neutropenia showed decreased granulopoiesis with no erythroid or megakaryocytic abnormalities. Cytogenetic analysis did not demonstrate abnormalities, and no anti-neutrophil antibodies were detected. Patient E1, the oldest patient in the series, had a hypocellular bone marrow with myeloid hypoplasia and mild megakaryocytic atypia; B cells and plasma cells were also drastically reduced (data not shown). Monocyte counts were normal in 5 of 7 patients (reduced in E1 and F1). Low myeloid as well as normal-low plasmacytoid DC numbers were observed in the 2 patients tested (B1 and G1) (Table 2 and Figure 4A). Targeted RNA-Seq was also done in LPS-stimulated monocytes from patients carrying IKZF1 ${ }^{\mathrm{N} 159 \mathrm{~S} / \mathrm{T}}$ mutants, healthy controls, and 2 patients carrying haploinsufficient mutation $\mathrm{IKZF1}^{\mathrm{H} 167 \mathrm{R}}$. Analysis of data revealed significantly differentially expressed transcripts between cells carrying mutants IKZF1 ${ }^{\mathrm{N} 159 \mathrm{~S} / \mathrm{T}}$ when compared with either healthy control or IKZF1 $1^{\mathrm{H} 167 \mathrm{R}}$ mono- 


\section{Table 2. Immunological features of PBMCs from patients with IKZF1 ${ }^{\mathrm{N} 1595 / \mathrm{T}}$ mutations $^{\mathrm{A}}$}

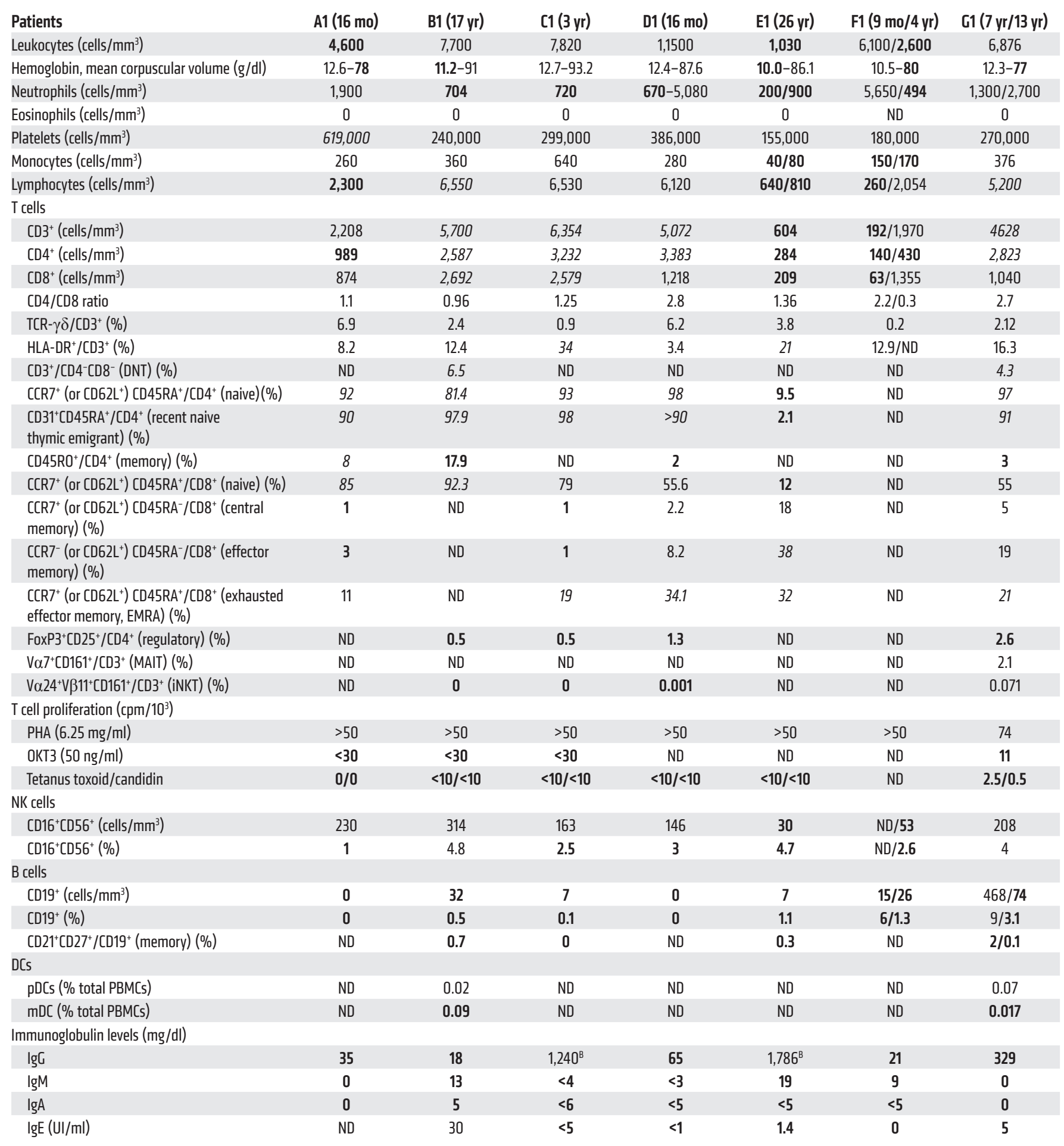

${ }^{A}$ Bold indicates below-normal values, and italics above-normal values, according to age-matched data $(44,45)$. ${ }^{8}$ On immunoglobulin replacement therapy. ND, not determined.

cytes. No differences were detected when healthy control and $\mathrm{IKZF}^{\mathrm{H} 167 \mathrm{R}}$ cells were compared with each other (Supplemental Figure 2B). In correlation with their abnormal transcript expression, monocyte proinflammatory cytokine production of IL-1 $\beta$,
IL-6, and TNF- $\alpha$ evaluated in 3 patients was severely impaired in response to agonists triggering Toll-like receptor $1 / 2$ (TLR1/2) (Pam3CSK4), TLR2 (HKLM), TLR4 (LPS), TLR5 (FLA-ST), and TLR6/2 (FSL1) pathways (Figure 4B), while adequate responses 
A

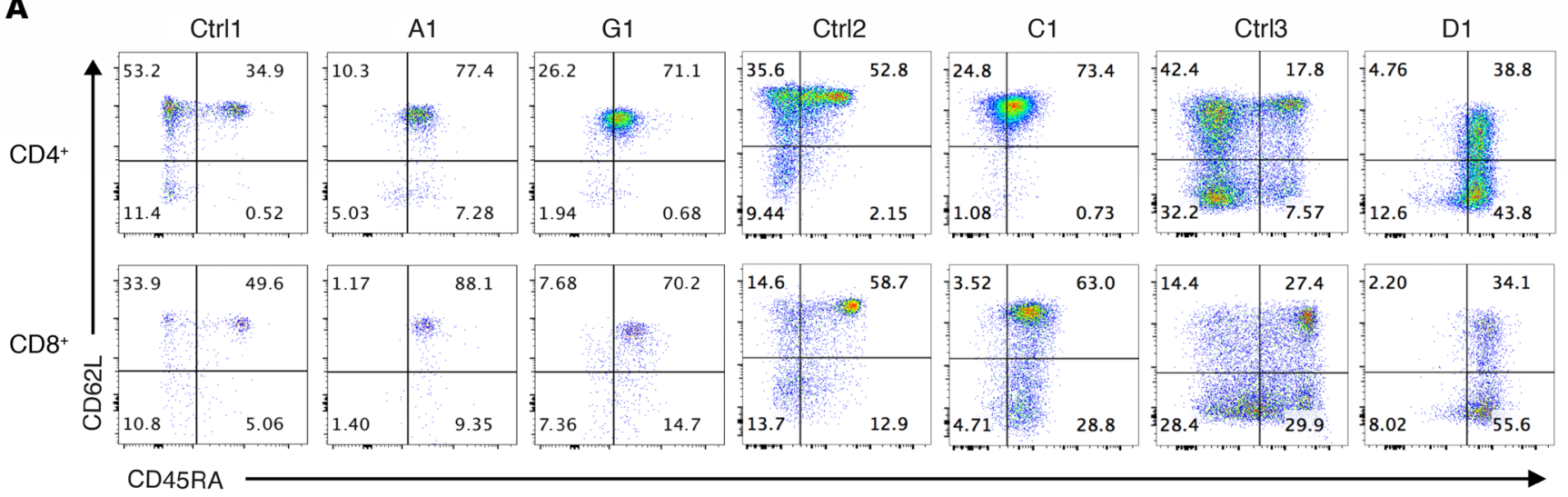

B
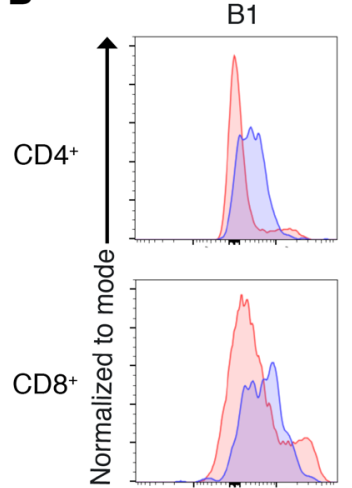

CD45RA
C1
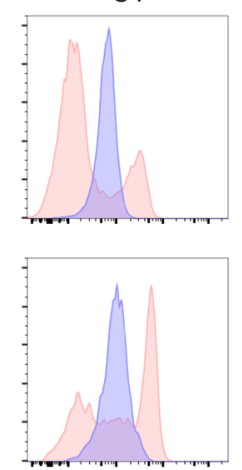

D1
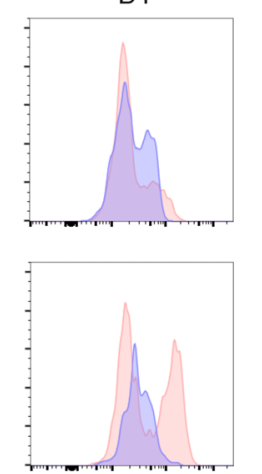

G1
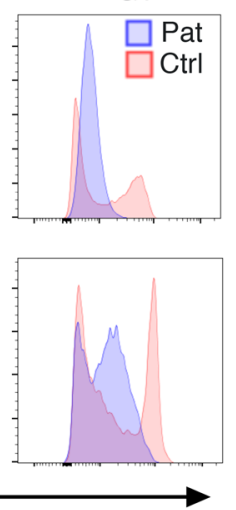

C

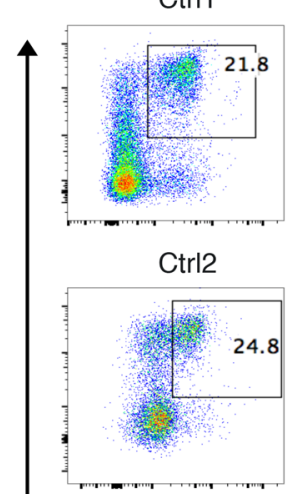

Ctrl3

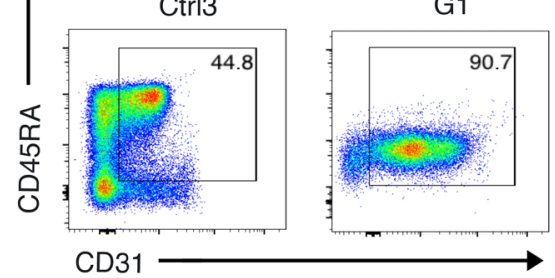

D

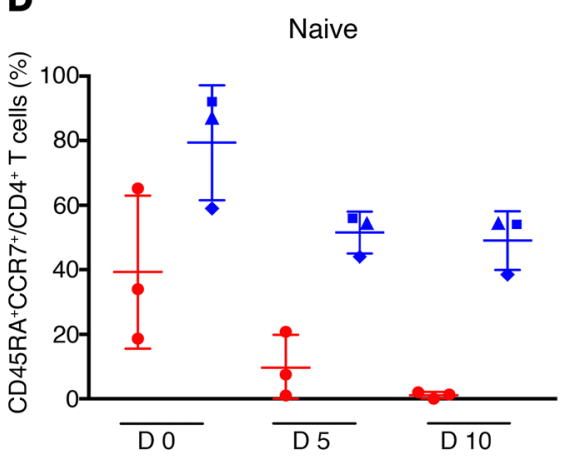

Central memory

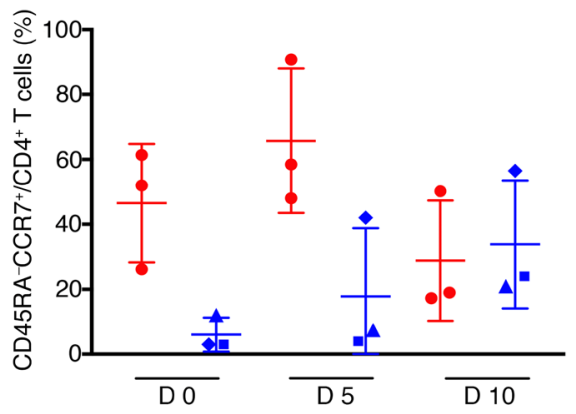

Effector memory

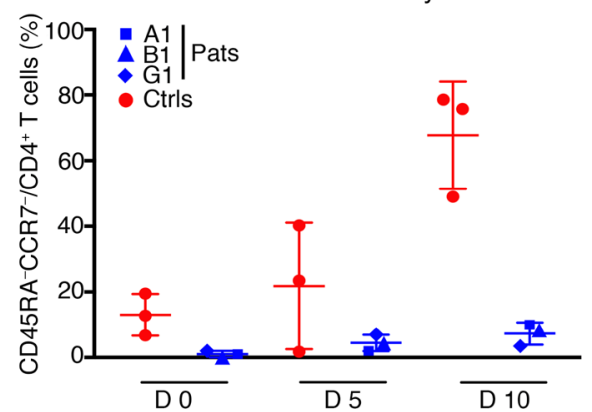

Figure 2. Peripheral T cell phenotypes associated with heterozygous IKZF1 ${ }^{11595 / T}$ mutations. (A) Dot plots from flow cytometric analyses showing

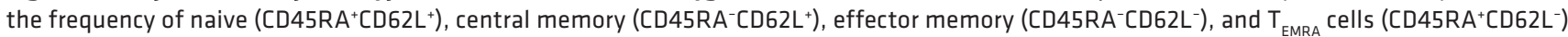
among $\mathrm{CD}^{+}$and $\mathrm{CD} 8^{+} \mathrm{T}$ cells of patients with $I K Z F 1^{\mathrm{N} 1595 / \mathrm{T}}$ mutations (A1, $\mathrm{G1}, \mathrm{C1}$, and $\mathrm{D1}$ ). Data are shown from independent experiments analyzed with a paired healthy control donor (Ctrl1, Ctrl2, and Ctrl3). (B) Histograms representing CD45RA staining of CD4 ${ }^{+}$and CD8 $8^{+}$T cells from patients (Pat: B1, C1, D1, and C1 in blue) overlaid with histograms from healthy donor controls (Ctrl in red). (C) Dot plots from flow cytometric analyses showing the frequency of recent thymic emigrants (CD45RA+CD31+) among CD4+ $\mathrm{T}$ cells from the indicated patient with a paired healthy donor control (Ctrl). (D) Evolution of naive (CD45RA $\left.{ }^{+} C C R 7^{+}\right)$, central memory (CD45RA-CCR7 ${ }^{+}$), and effector memory cells (CD45RA-CCR7-) in percentages (\%) of PHA-derived $\mathrm{CD}^{+} \mathrm{T}$ cell blasts cultured for 10 days from PBMCs of patients (Pats: A1, B1, and G1) and 3 healthy donor controls (Ctrls). Data represent mean \pm SD of 3 independent experiments. 
to IFN- $\alpha$ and IFN- $\gamma$ (by means of STAT1 phosphorylation) or IL-4 (evaluated by STAT6 phosphorylation) were detected (data not shown). Finally, addition of healthy control monocytes or B cells completely or partially restored (respectively) the proliferation defect of T cells from PBMCs of 2 IKZF1 ${ }^{\mathrm{N} 1595}$ patients when stimulated with soluble anti-CD3/CD28. This suggests that functionally defective monocytes and/or the absence of B cells in IKZF1 ${ }^{\text {N159s }}$ patients contribute to the $\mathrm{T}$ cell proliferation defect (Figure 4C).

Collectively, these observations indicate that heterozygous mutations yielding IKZF1 ${ }^{\mathrm{N} 159 \mathrm{~S} / \mathrm{T}}$ are associated not only with a CID affecting $\mathrm{B}$ and $\mathrm{T}$ cell function, but also with myeloid abnormalities suggestive of a multilineage hematopoietic defect. Myeloid defects could also directly contribute to the $\mathrm{T}$ cell defects in vivo in the patients, as addition of monocytes from healthy donors rescued $\mathrm{T}$ cell proliferative response from PBMCs of patients in vitro. This defective $\mathrm{T}$ cell proliferative response might be the result of impaired cytokine production by monocytes and/or antigen presentation, both involved in $\mathrm{T}$ cell activation, in addition to the already-established intrinsic T cell defects demonstrated in these patients.

Importantly, the clinical and biological picture associated with IKZF1 $^{\mathrm{N} 159 \mathrm{~S}}$ and IKZF1 ${ }^{\mathrm{N} 159 \mathrm{~T}}$ mutations, including the susceptibility to T-ALL development, strongly contrasts with the B cell-centric deficiency caused by previously reported haploinsufficient IKZF1 mutations $(2,18,19,22)$. Therefore, we hypothesized that mutations IKZF1 $1^{\mathrm{N} 159 \mathrm{~S}}$ and IKZF1 ${ }^{\mathrm{N} 159 \mathrm{~T}}$ may act through a different mechanism than haploinsufficiency.

Dominant-negative behavior of IKZF1 ${ }^{\mathrm{N} 159 \mathrm{~S}}$ and IKZF1 ${ }^{\mathrm{N} 159 \mathrm{~T}}$ mutations. Expression levels and functional effects of mutant proteins IKZF1 $^{\mathrm{N} 159 \mathrm{~S}}$ and IKZF1 ${ }^{\mathrm{N} 159 \mathrm{~T}}$ were assessed in patients' cells as well as in vitro expression experiments. IKZF1 expression was evaluated by means of flow cytometry (PBMCs from patients C1 and D1) and Western blot analysis using $\mathrm{T}$ cell blasts from patient G1 (Figure $5 \mathrm{~A})$. No protein expression differences were noticed between cells from patients and healthy controls. Mutant proteins IKZF1 ${ }^{\mathrm{N} 1595}$ and IKZF1 ${ }^{\mathrm{N} 159 \mathrm{~T}}$ were expressed in transiently transfected HEK293T cells and compared with WT IKZF1 or with the previously described haploinsufficient IKZF1 ${ }^{\mathrm{R} 162 \mathrm{Q}}$ mutant protein. All proteins were found to be expressed at similar levels, indicating that N159S and N159T mutants are expressed and stable (Figure $5 \mathrm{~B}$ ). Binding of WT and mutant IKZF1 proteins (alone or in combination) to the PC-HC sequences of the $\gamma$ satellite region of chromosome 8 ( $\gamma$-Sat8) or IKAROS consensus-binding sequence 4 (IK-bs4) was evaluated by electrophoretic mobility shift assay (EMSA) using protein extracts from HEK293T transfected cells. None of the 3 mutant proteins were able to bind to $\gamma$-Sat 8 or IK-bs 4 , and $40 \%-50 \%$ reduction in binding was evidenced in the 50:50 mutant/WT protein combination tested (Figure 5, C and D).

The function of IKZF1 ${ }^{\mathrm{N} 159 \mathrm{~S}}$ and IKZF1 ${ }^{\mathrm{N} 159 \mathrm{~T}}$ proteins was assessed by their ability to dimerize, migrate to the nucleus, and form foci by binding to PC-HC, as previously reported $(14,21)$. Mutant proteins IKZF1 $^{\text {N159S }}$ and IKZF1 ${ }^{\text {N159T }}$ were able to dimerize with WT IKZF1 (Figure 5E) and migrate to the nucleus (Figure 6A and Supplemental Figure 6). IKZF1 ${ }^{\mathrm{N} 159 \mathrm{~S}}$, IKZF1 ${ }^{\mathrm{N} 159 \mathrm{~T}}$, and IKZF1 ${ }^{\mathrm{R} 162 \mathrm{Q}}$ were transiently expressed alone or with WT IKZF1 in NIH 3T3 cells at different ratios, and formation of foci was examined at the single-cell level (Figure 6). At 25:75, 50:50, and 75:25 mutant/WT ratios, coexpres- sion of IKZF1 ${ }^{\mathrm{N} 159 \mathrm{~S}}$ or IKZF1 ${ }^{\mathrm{N} 159 \mathrm{~T}}$ with WT IKZF1 showed a significant decrease in IKZF1 foci per nucleus, in comparison to the number of foci detected in cells transfected with WT IKZF1 alone (0:100 ratio) or in 50:50 combination with IKZF1 ${ }^{\mathrm{R} 162 \mathrm{Q}}$ (Figure 6, B and C). Experiments using tagged forms of mutants and WT IKZF1 showed similar results (Supplemental Figure 6). At the 25:75 ratio, mutant $\mathrm{IKZF}^{\mathrm{N} 159 \mathrm{~T}}$ formed more foci than IKZF1 ${ }^{\mathrm{N} 159 \mathrm{~S}}$ (Figure 6C). Coexpression of IKZF1 ${ }^{\mathrm{N} 159 \mathrm{~S}}$ or IKZF1 ${ }^{\mathrm{N} 159 \mathrm{~T}}$ with IKZF3 (AIOLOS) in a 50:50 ratio resulted in heterodimerization and inhibition of IKZF3 foci formation associated with diffused nuclear localization of IKZF3, similar to what was seen with IKZF1 (Supplemental Figure 7).

These data demonstrate that IKZF1 $1^{\mathrm{N} 159 \mathrm{~S}}$ and IKZF1 ${ }^{\mathrm{N} 159 \mathrm{~T}}$ mutant proteins are expressed, and can dimerize and migrate to the nucleus, where they exert a dominant-negative effect on WT IKZF1, in terms of formation of foci within the nucleus, in contrast to the findings of the previously described haploinsufficient IKZF1 mutants (e.g., IKZF1 $1^{\mathrm{R} 162 \mathrm{Q}}$ ). Of note, the IKZF1 ${ }^{\mathrm{N} 159 \mathrm{~S}}$ mutant exhibited a stronger dominant-negative effect on formation of foci than the IKZF1 ${ }^{\mathrm{N} 159 \mathrm{~T}}$, in accordance with the clinical and biological presentations of the IKZF1 ${ }^{\mathrm{N} 159 \mathrm{~T}}$ patient, which are less pronounced. Finally, $\mathrm{IKZF}^{\mathrm{N} 159 \mathrm{~S}}$ and IKZF1 ${ }^{\mathrm{N} 159 \mathrm{~T}}$ can also exert a trans-dominant-negative effect on IKZF1 partners such as IKZF3.

\section{Discussion}

Herein we describe a new autosomal dominant CID caused by IKZF1 $1^{\mathrm{N} 159 \mathrm{~S} / \mathrm{T}}$ mutations found in 7 unrelated patients. This disorder is characterized by a $\mathrm{T}$ cell defect affecting activation, signaling, proliferation, T-helper (Th) polarization, and memory generation, with susceptibility to Pneumocystis pneumonia prior to the age of 2 years. This condition, similar to the previously described IKZF1 haploinsufficiency defect, is also associated with impaired B cell development and maintenance, resulting in loss of B cells in most cases. Additionally, there are myeloid abnormalities including neutropenia, eosinopenia, quantitative defects in myeloid DCs, and abnormal monocyte function that likely contribute to the $\mathrm{T}$ cell defect. All these findings are consistent with hematopoietic pan-expression of IKZF1. This defect also likely predisposes to an increased risk of T-ALL, as was previously reported in 1 patient in our series (2).

Besides $P$. jirovecii pneumonia, most patients also developed severe respiratory bacterial (pneumococci, klebsiella, and pseudomonas) or viral (respiratory syncytial virus [RSV], influenza $\mathrm{A}$, and adenovirus) infections. Interestingly, other microbes appeared to be better neutralized among IKZF1 N159/T patients. While 2 individuals received live viral vaccines (either measles-mumps-rubella [MMR] or oral poliovirus vaccine) and 3 were bacillus Calmette-Guérin (BCG) vaccinated, neither of them experienced complications related to the vaccines. Moreover, 2 patients presented with chickenpox with uncomplicated courses. Several factors might have contributed to this protection toward particular infectious agents. Although these patients exhibited marked $\mathrm{T}$ cell defects, $\mathrm{T}$ cell proliferative responses to high-dose CD3 or to costimulation with CD3/CD28-coated beads were normal. This residual proliferation capacity upon stronger stimulation might be sufficient to control certain infections. Moreover, normal responses to type I and type II interferons were detected in these patients (data not shown), both critical for the control of viral and 


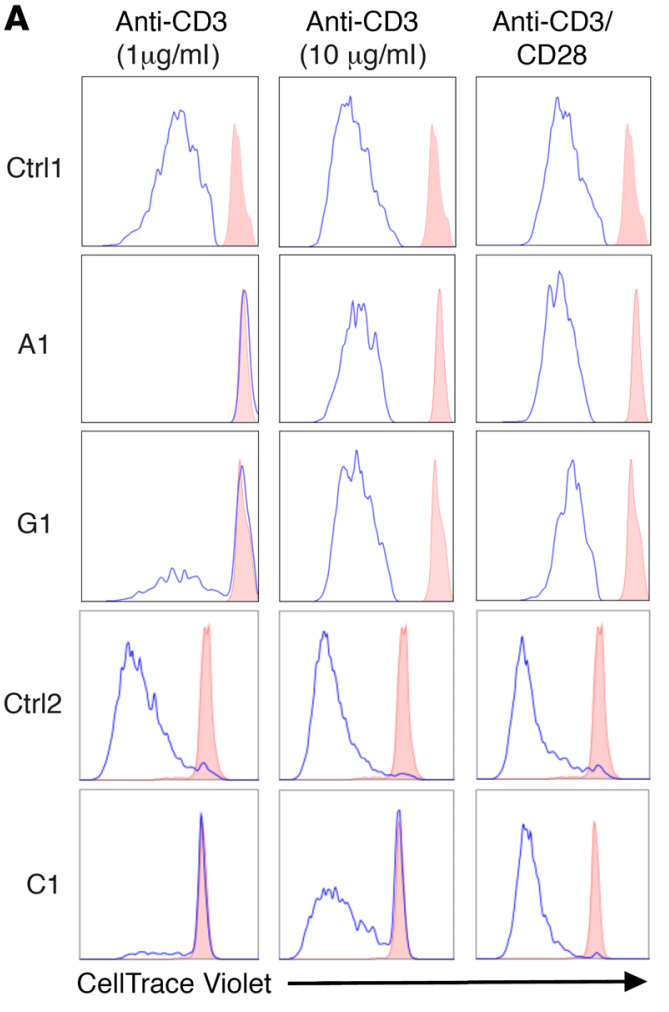

B

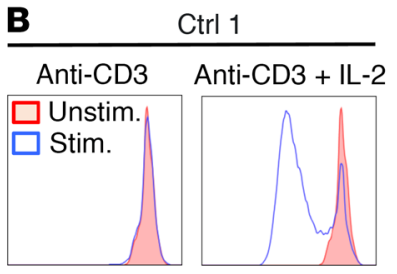

CellTrace Violet

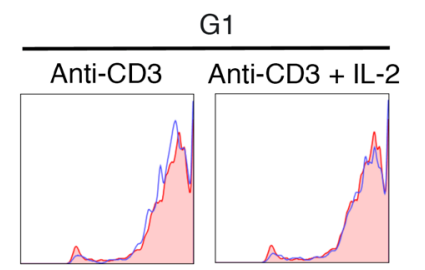

CellTrace Violet
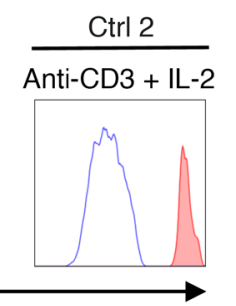

A1

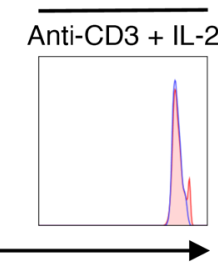

C

$$
\begin{aligned}
& \text { Unstim. } \\
& \text { - Anti-CD3 }(1 \mu \mathrm{g} / \mathrm{ml}) \\
& \text { - Anti-CD3 }(10 \mu \mathrm{g} / \mathrm{ml}) \\
& \text { - Anti-CD3 }(0.05 \mu \mathrm{g} / \mathrm{ml})+\mathrm{IL}-2
\end{aligned}
$$
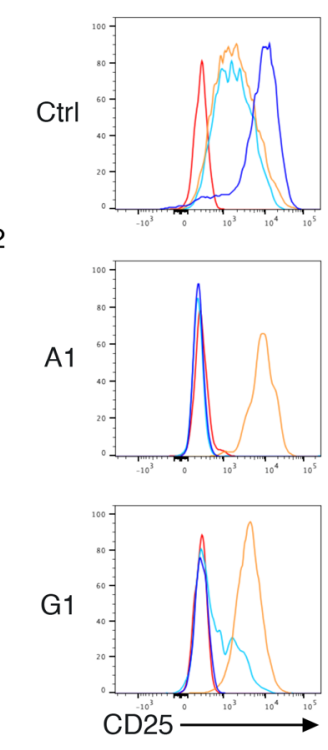

D
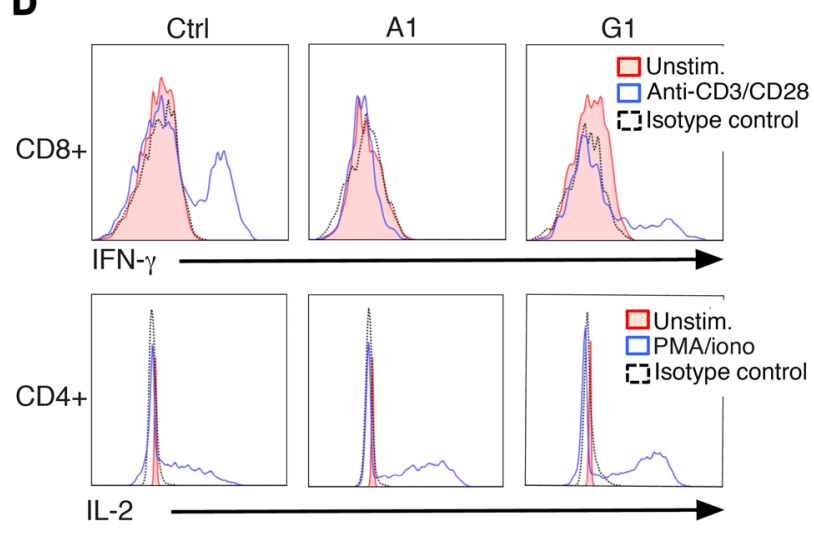

E
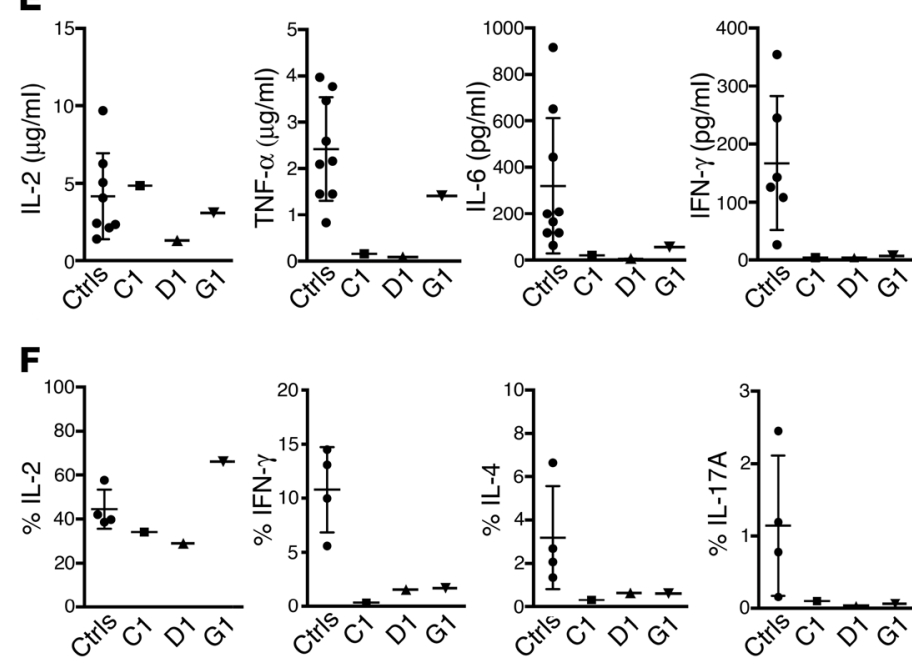

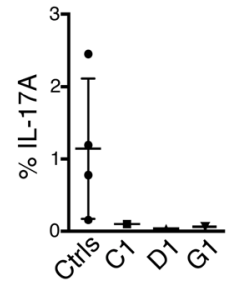

Figure 3. Functional T cell defects associated with heterozygous IKZF1 ${ }^{N 1595 / T}$ mutations. (A-C) PBMCs from patients $A 1, C 1$, and $C 1$ and healthy donor controls (Ctrl) were stimulated with indicated agonists for 6-7 days. (A) Cells stimulated (stim.) with plate-coated anti-CD3 at the indicated concentrations or with anti-CD3/CD28-coated beads. (B) Cells stimulated with plate-coated anti-CD3 at $0.05 \mu \mathrm{g} / \mathrm{ml}$ with or without IL-2. unstim., unstimulated. (C) Cells stimulated with plate-coated anti-CD3 at the indicated concentrations with or without IL-2. Proliferation was determined by dilution of CellTrace Violet dye analyzed by flow cytometry. Histograms showing cell divisions by dilution of the CellTrace Violet dye (A and B) and CD25 expression (C). Data are representative of 3 experiments. (D) Histograms from PHA-derived T cell blasts from patients A1, G1, and a healthy donor control (Ctrl) stimulated with (blue) or without (red) anti-CD3/CD28-coated beads (upper panels) or with PMA/ionomycin (iono; lower panels) for 24 hours. IL-2 (lower panels) and IFN- $\gamma$ (upper panels) were measured with intracellular flow cytometry assays. Black dashed line indicates isotype control. Data are representative of 2 experiments. (E) PBMCs from patients $\mathrm{C1}, \mathrm{D} 1$, and $\mathrm{C} 1$ and healthy donor controls (Ctrls) were stimulated with anti-CD3/CD28-coated beads for 24 hours. Cell-free supernatants were harvested, and cytokines (IL-2, TNF- $\alpha$, IL-6, and IFN- $\gamma$ ) were measured with the Luminex 200 System. Data indicate the mean of replicate sample for $\mathrm{C} 1(n=2)$, D1 $(n=1)$, and $\mathrm{C1}(n=1)$ compared with 6-9 different healthy donor controls (mean \pm SD). The $n$ values represent the number of replicates. (F) PBMCs from patients C1, D1, G1, and 2 paired healthy donor controls (Ctrls) were stimulated with PMA/ionomycin for 6 hours. IL-2, IFN- $\gamma$, and IL-4 (for Th1/2 evaluation) and IL-17A (for Th17 evaluation) were measured with intracellular flow cytometry assays. Data represent the percentage of the cells positive for the indicated cytokine among $\mathrm{CD} 4^{+} \mathrm{T}$ cells. Data are issued from 2 experiments performed in patient $\mathrm{C} 1$ and 1 experiment from $\mathrm{D} 1$ and $\mathrm{C} 1$. 
mycobacterial infections (respectively) (23-25). All together, these preserved mechanisms might help to reduce the spectrum and clinical severity of certain infectious diseases in these individuals. Of note, autoimmunity and/or atopy were not detected in these patients despite low regulatory $\mathrm{T}$ cell numbers or/and increased DNT cell values. The drastic decrease in B cell and plasma cell numbers, as well as memory $\mathrm{T}$ cell numbers, together with defective Th2 polarization, eosinopenia, and low-to-undetectable levels of IgE, might prevent the expression of such symptoms.

This novel CID displayed a complex T cell deficiency. First, most of the $\mathrm{T}$ cells were of a naive Th0-like phenotype but uniformly showed a decreased level of CD45RA expression associated with a recent thymic emigrant (RTE) phenotype (CD31+). These features may be useful for screening patients with CID in settings where a dominant-negative $I K Z F 1$ defect is suspected. T cell receptor excision circles (TRECs) were evaluated in 3 patients to further confirm the RTE status of these cells (26). TRECs were in normal range in 2 patients and elevated in 1 patient (Supplemental Figure 2C), which suggests that CD31 expression on naive T cells did not completely correlate with an RTE phenotype in our patients. Thus, CD31 dysregulation might be part of the defects associated with IKZF1 $1^{\mathrm{N} 159 \mathrm{~N} / \mathrm{T}}$ mutations. Furthermore, transcriptome analysis of $\mathrm{CD}^{+}$naive $\mathrm{T}$ cells $\left(\mathrm{CD}^{+} \mathrm{CD} 4^{+} \mathrm{CD} 45 \mathrm{RA}^{+}\right)$from patients with IKZF1 ${ }^{\mathrm{N} 159 \mathrm{~S} / \mathrm{T}}$ mutations showed striking differences in terms of up- and downregulated genes when compared with $\mathrm{CD}^{+}$naive $\mathrm{T}$ cells from healthy controls and a patient carrying haploinsufficient mutation IKZF1 $1^{\mathrm{H} 167 \mathrm{R}}$ (Supplemental Figure 2A). These data confirmed that circulating naive T cells in IKZF1 ${ }^{\mathrm{N} 159 \mathrm{~S} / \mathrm{T}}$ patients are abnormal and are also different from those in patients carrying haploinsufficiency mutations. These results could be indicative of abnormal lymphopoiesis, which may contribute to the risk for T-ALL in patients carrying dominant-negative IKZF1 $1^{\mathrm{N} 159 \mathrm{~N} / \mathrm{T}}$ mutations, as they are reminiscent of the early $\mathrm{T}$ cell development abnormalities increasing the risk for $\mathrm{T}$ cell leukemia/lymphoma as seen in the Plastic/+ mice also carrying a dominant-negative heterozygous missense mutation $(14,15)$. Interestingly, TCR-V $\beta$ flow cytometric repertoire and TCR-V $\alpha$ spectratyping showed a normal $\mathrm{T}$ cell distribution, which suggests that $\mathrm{T}$ cell thymic education was not obviously impacted in IKZF1 ${ }^{\mathrm{N} 159 \mathrm{~S} / \mathrm{T}}$ patients (data not shown). Further studies are warranted in order to better characterize these $\mathrm{T}$ cells. As with $\mathrm{CD} 4^{+}$naive $\mathrm{T}$ cells, transcriptome analysis of monocytes from IKZF1 ${ }^{\mathrm{N} 159 \mathrm{~S} / \mathrm{T}}$ patients also revealed marked differences when compared with healthy controls or the haploinsufficient IKZF1 ${ }^{\mathrm{H} 167 \mathrm{R}}$ mutated patients (Supplemental Figure 2B). Altogether these data correlate with the lack of abnormal $\mathrm{T}$ cell or monocyte phenotype in the IKZF1-haploinsufficient patients.

Second, peripheral blood $\mathrm{T}$ cells exhibited variably impaired proliferation defects depending on the stimulus and decreased IFN- $\gamma$, TNF- $\alpha$, IL-4, IL-6, and IL-17A production indicative of a global defective Th polarization, in which cells seem arrested in a Th0-like phenotype. The observation that $\mathrm{T}$ cell proliferation was abnormal in response to recall antigens or to low concentrations of anti-CD3 suggests the possibility of an additional defect affecting accessory cells. Consistent with this hypothesis were the findings of low numbers of myeloid DCs and impaired cytokine production by monocytes in response to several TLR agonists. Furthermore, total or partial correction of $\mathrm{T}$ cell proliferation was observed after addition of either healthy control monocytes or B cells, respectively. This finding could have relevant clinical implications in patients undergoing HSCT, as lymphoid engraftment alone or mixed chimerism may not suffice to rescue the phenotype. The $\mathrm{T}$ cell proliferation defect was also associated with an impaired CD25 upregulation after TCR engagement, which could explain why IL-2 did not rescue the defective $\mathrm{T}$ cell proliferation. However, all these features may also be in part secondary to a $\mathrm{T}$ cell developmental defect resulting in hyporeactive $\mathrm{T}$ cells with low-density CD45RA expression (27). Importantly, 1 patient (G1) had preserved memory $\mathrm{T}$ cells but still exhibited the same $\mathrm{T}$ cell proliferation and Th polarization defect, which suggests that the absence of memory $\mathrm{T}$ cells in the other patients was not solely responsible for this functional defect.

Third, as opposed to most forms of CID, which are characterized by a depletion in naive and accumulation of memory $\mathrm{T}$ cells (28), there was a profound defect in memory $\mathrm{T}$ cell acquisition and maintenance both in vivo and in vitro, which points toward a specific role for IKZF1 in the development of memory T cells. Interestingly, this particular feature was almost exclusively described to be associated with genetic defects leading to loss of function of NF- $\mathrm{BB}$ activation, such as IKBA, IKBKB, CARMIL2, or BCM (BCL1O, CARMA1, and MALT1) deficiencies (29-31). We therefore evaluated the NF- $\kappa \mathrm{B}$ canonical pathway (by means of I $\mathrm{B} \alpha$ degradation and p65 phosphorylation in 2 patients, C1 and G1) and did not find any evidence for abnormal NF- $\kappa \mathrm{B}$ signaling (not shown). These findings do not exclude a more downstream signaling defect at the DNA-binding level or other NF- $\kappa \mathrm{B}$ regulatory defects. Indeed, Trung et al. (32) have recently shown that lymphoid hyperconserved core promoter-associated M4 motifs could constitute a binding platform for IKZF1 and NFкB1, with optimal signaling when both proteins are present.

The spectrum of manifestations associated with IKZF1 ${ }^{\mathrm{N} 159 \mathrm{~S} / \mathrm{T}}$ also include various myeloid defects. While eosinopenia was present in all patients tested, neutropenia was detected in most but not all affected individuals, imposing an increased risk factor for infections. Even if monocyte counts remained normal, in vitro functional evaluation demonstrated a severe impairment in the production of cytokines in response to TLR1, TLR2, TLR4, TLR5, and TLR6 stimulation, and transcriptome analysis revealed an abnormal phenotype. In addition, myeloid DCs were severely diminished in the patients tested. This latter finding could be involved in Cryptosporidium susceptibility, as was diagnosed in 1 patient (A1). Cryptosporidiosis is associated with a restricted number of primary immune deficiencies, including CD40 ligand (33), MHC class II (34), and IL-21 receptor deficiencies (35), all defects characterized by impaired crosstalk between $\mathrm{T}$ cells and antigen-presenting cells. MHC class II expression was normal in all patients tested. CD4OL upregulation and CD40 binding were normal in 3 patients (C1, D1, and G1) but reduced in 1 (A1) (data not shown) who presented with cryptosporidiosis. As CD40L expression was evaluated after standard PMA/ionomycin stimulation, we cannot exclude that under physiologic $\mathrm{T}$ cell activation conditions a more consistent CD4OL upregulation defect could be unveiled (as shown with CD25) and therefore contribute to increased Pneumocystis and Cryptosporidium susceptibility. The same rationale applies to the role of IL-21 signaling (not tested). 

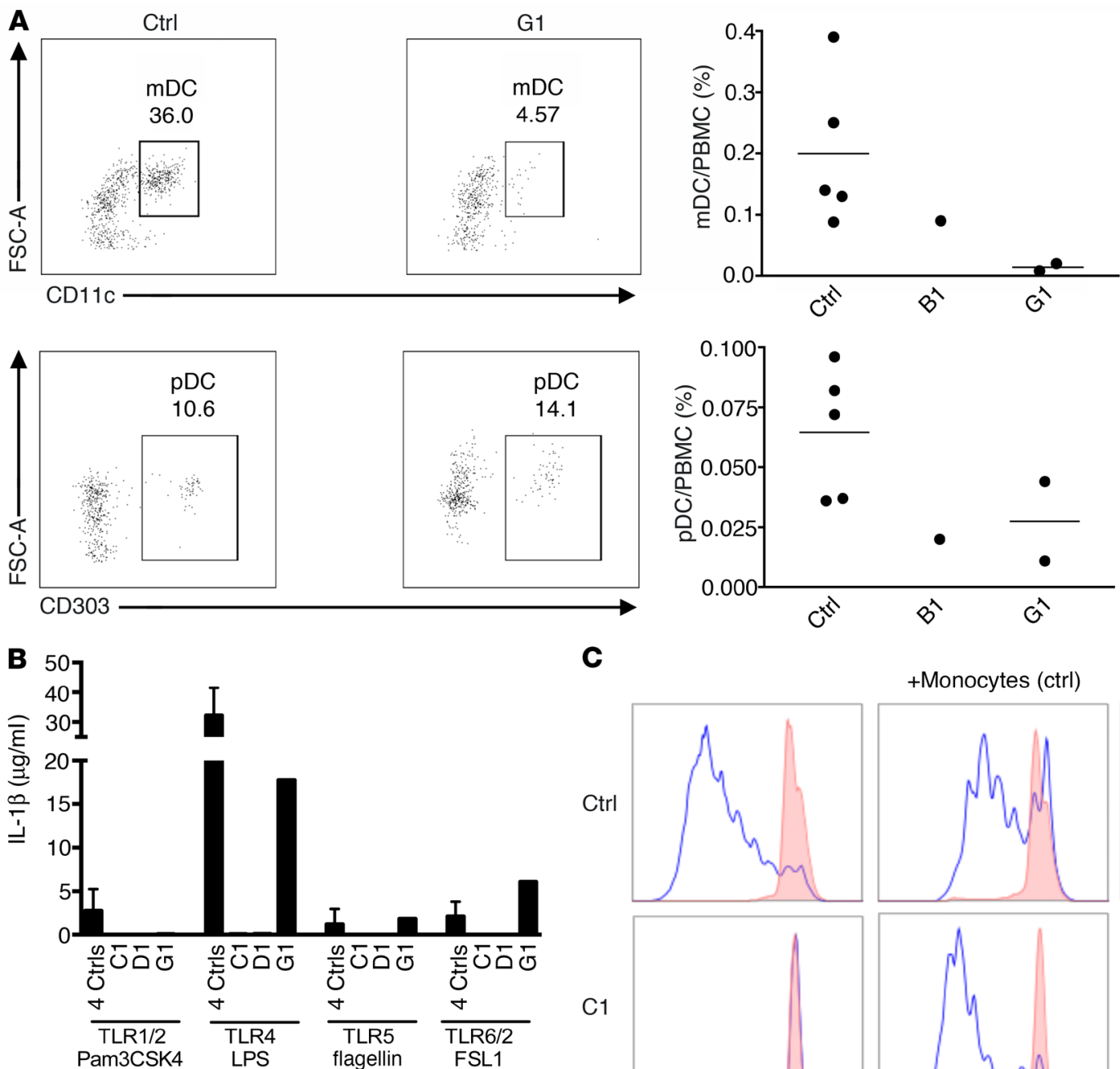

C

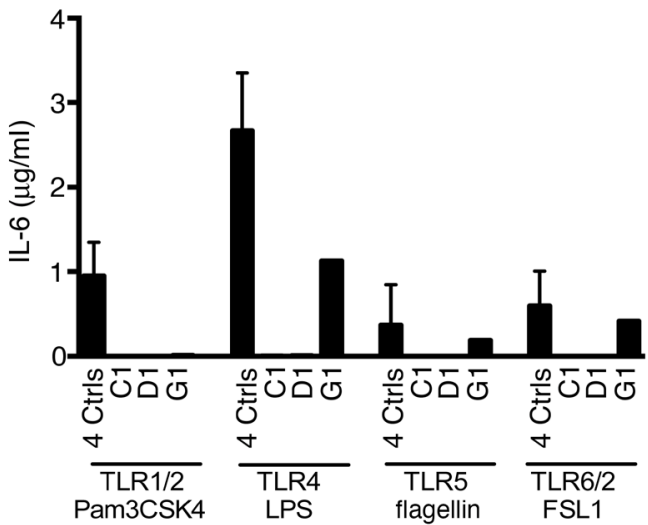

C1

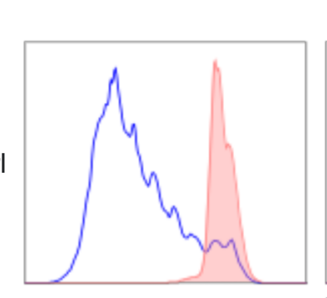

+Monocytes (ctrl)

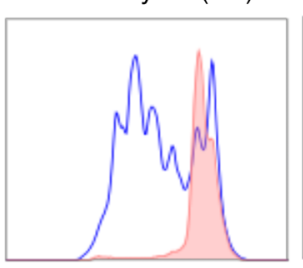

$+\mathrm{B}$ cells (ctrl)
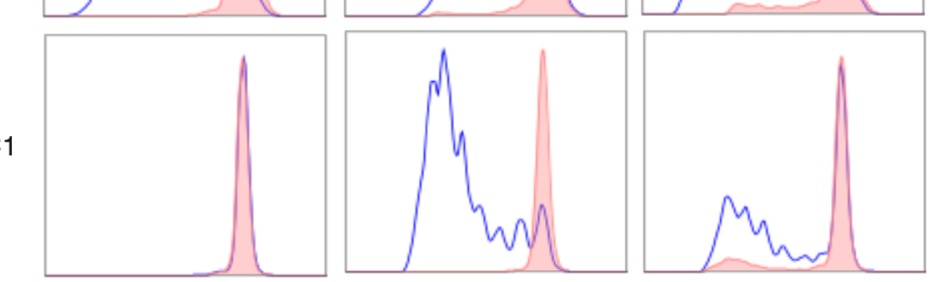

D1

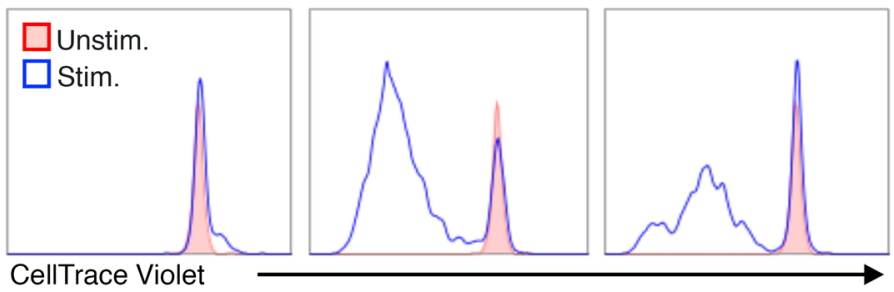

Figure 4. Myeloid abnormalities associated with heterozygous IKZF1 ${ }^{\mathrm{N} 1595 / \mathrm{T}}$ mutations. (A) PBMCs from G1 and a healthy donor control (Ctrl) were analyzed for the presence of DCs. Dot plots (left panels) of lineage-negative (CD3-, CD19-' and $\left.\mathrm{CD}^{-} 6^{-}\right)$, HLA-DR-positive, and CD14- or CD16- negative population corresponding to DCs. The CD11c and CD303 markers enabled the delineation of mDCs

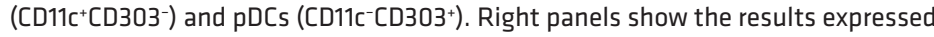
as percentage of PBMCs from patients B1, $\mathrm{C} 1$, and 5 healthy donor controls. (B) PBMCs from C1, D1, and C1 and healthy donor controls (Ctrl) were stimulated with the indicated TLR agonist for 24 hours. Cell-free supernatants were harvested, and cytokines (IL-6, IL-1 $\beta$, TNF- $\alpha$ ) were measured with the Luminex 200 System. Data are from one experiment performed in patients $\mathrm{C} 1, \mathrm{D} 1$, and $\mathrm{C} 1$ and compared with 4 healthy donor controls (mean \pm SD). (C) PBMCs from C1 and D1 were stimulated with (blue line) or without (solid red) soluble anti-CD3 and anti-CD28 antibodies $(1 \mu \mathrm{g} / \mathrm{ml}$ each) in the presence or absence of the healthy donor's monocytes or $\mathrm{B}$ cells for 5 days. The proliferation was determined by dilution of CellTrace Violet dye analyzed by flow cytometry. Data are representative of 3 experiments. 
A

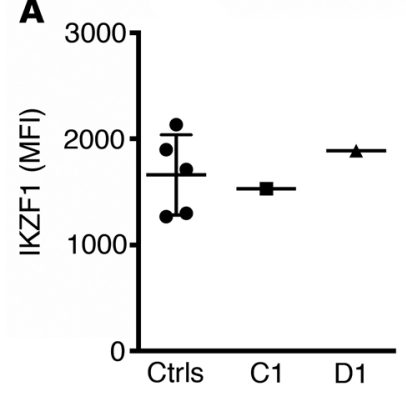

D
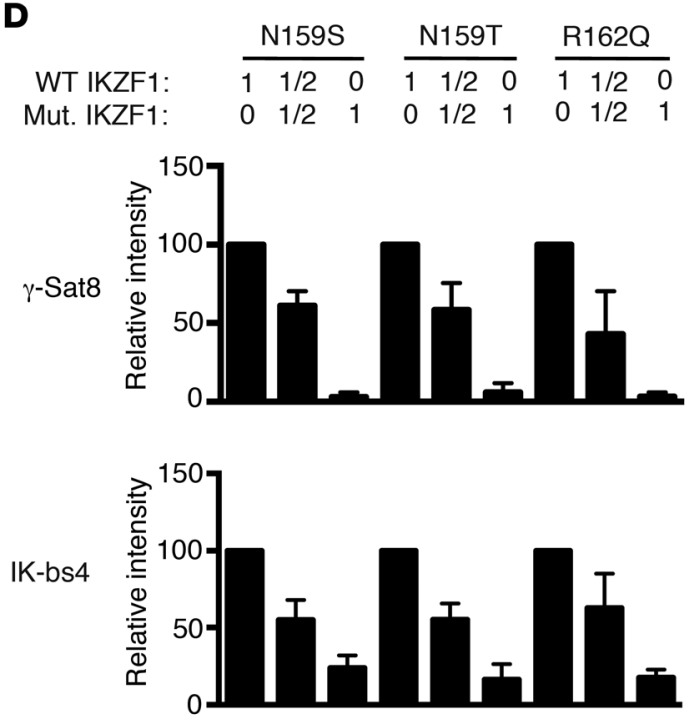

B

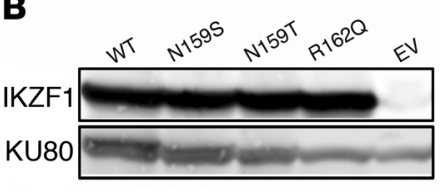

C
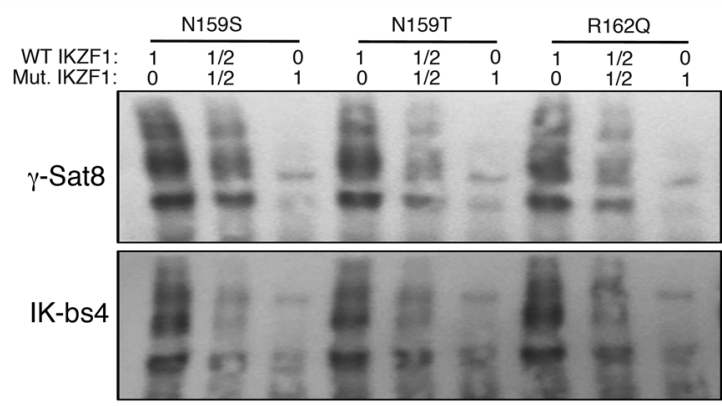

Anti-IKZF1

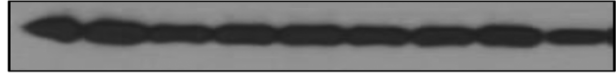

E

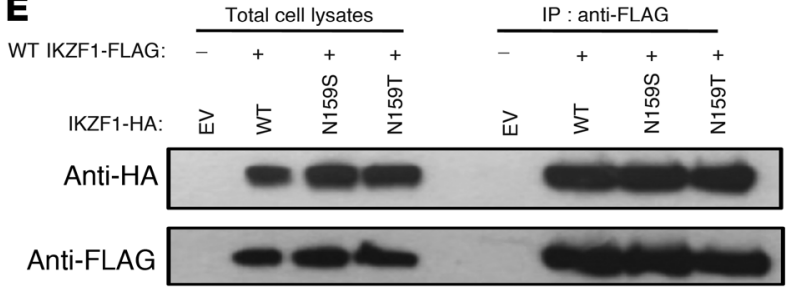

Figure 5. Expression, dimerization, and DNA binding of IKZF1 ${ }^{\mathrm{N} 1595 / T}$ mutants. (A) IKZF1 expression was evaluated in 3 patients (C1, D1, and G1). Permeabilized $\mathrm{CD}^{+} \mathrm{T}$ cells of $\mathrm{C} 1$ and $\mathrm{D} 1$ were stained with PE-conjugated anti-IKZF1 antibodies and results expressed as mean fluorescence intensity (MFI) from FACS analysis (left panel). Data are mean \pm SD of 5 healthy donor controls paired with the indicated patients. Cell extracts of PHA-derived T cell blasts of G1 were immunoblotted with anti-IKZF1 or anti-KU80 antibodies as a loading control (right panel). (B) Expression of IKZF1 in HEK293T cells transiently transfected with WT IKZF1, IKZF1 mutants (N159S, N159T, R162Q), or empty vector (EV). IKZF1 ${ }^{\text {R1620 }}$ haploinsufficient mutant was used for comparison. Cell lysates were immunoblotted with anti-IKZF1 or anti-KU80 antibodies as a loading control. (C) EMSAs were performed with nuclear extracts from HEK293T cells transiently transfected with WT IKZF1 and/or the indicated IKZF1 mutants (FLAG-tagged IKZF1). IKZF1 ${ }^{11620}$ haploinsufficient mutant was used for comparison. The nuclear extracts were tested by gel mobility shift assay for binding to $\gamma$-Sat8 (upper panel) and IK-bs4 (middle panel), 2 sequences from pericentromeric regions known to be IKZF1 targets (upper panels). Cell lysates were tested by Western blot for WT and/or mutant IKZF1-FLAG expression with an anti-IKZF1 antibody (lower panel). Data shown are representative of 3 experiments. (D) EMSA bands were quantified by the Bio-Rad Image Lab program. Data were normalized to WT binding defined as 100\%. Error bars represent SD from 3 independent experiments. (E) Cell lysates of HEK293T cells transiently transfected with WT IKZF1-FLAG and WT or mutant IKZF1-HA were immunoprecipitated (IP) with anti-FLAG antibody. Data represent Western blot of whole cell lysates and IP samples (right panel) with anti-HA (upper right panels) and anti-FLAC antibodies (lower right panels). Whole cell lysates correspond to $5 \%$ of proteins used in IP (left panel). Data shown are representative of 3 experiments.

Interestingly, patient E1 was first suspected to have GATA2 deficiency based on bone marrow hypocellularity, monocytopenia, and the complete absence of B cells. Haploinsufficiency of GATA2, another transcription factor expressed in hematopoietic cell progenitors, is a monogenic dominant immune deficiency leading to myeloid and lymphoid defects and increased susceptibility to hematologic malignancies (36), characteristics akin to those of the CID caused by IKZF1 ${ }^{\mathrm{N} 1598 / \mathrm{T}}$ mutations described herein. Interestingly, a crosstalk between Ikaros and Gata2 transcription factors was clearly established in mouse models, as Ikaros expression is differentially regulated by Gata2 and Gata1 during megakaryocytic development (7).

Patients with somatic and germline IKZF1 mutations are associated with increased susceptibility to and poor outcome from selected lymphoid malignancies including ALL. In particular, the somatic mutation IKZF $1^{\mathrm{N} 159 \mathrm{~S}}$ has been found in patients with B-ALL (37). Our study suggests that carriers of germline IKZF1 $1^{\mathrm{N} 159 \mathrm{~s}}$ mutation likely have an increased risk of developing T-ALL. Thus, we searched for IKZF1 ${ }^{\mathrm{N} 159 \mathrm{~S}}$ or IKZF1 ${ }^{\mathrm{N} 159 \mathrm{~T}}$ mutations in a cohort of patients with T-ALL, for which tumor material had been analyzed by next-generation sequencing. One tumor sample was found to contain a somatic heterozygous mutation, IKZF1 ${ }^{\mathrm{N} 159 \mathrm{~S}}$, and was not detected in the PBMCs of the patient after induction of remission, which ruled out that the mutation was germinal, and $100 \%$ of leukemic cells were found to carry the IKZF1 ${ }^{\mathrm{N} 159 \mathrm{~S}}$ mutation (D. Boutboul, S. Latour, L. Lhermitte and V. Asnafi, unpublished observations). Altogether, these observations indicate that mutation IKZF1 ${ }^{\mathrm{N} 159 \mathrm{~S}}$ can be a leading genetic event associated with leukemogenesis, suggesting that there may be a phenotypic continuum between somatic and germline IKZF1 ${ }^{\mathrm{N} 159 \mathrm{~s}}$ mutations. 
A
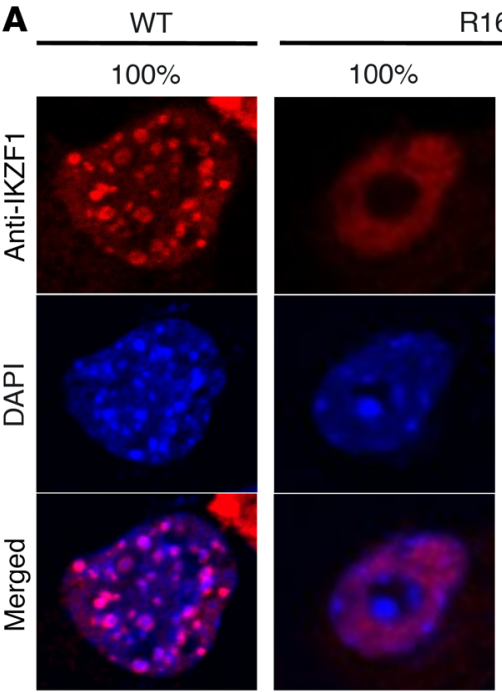

R162Q

$+50 \% \mathrm{WT}$
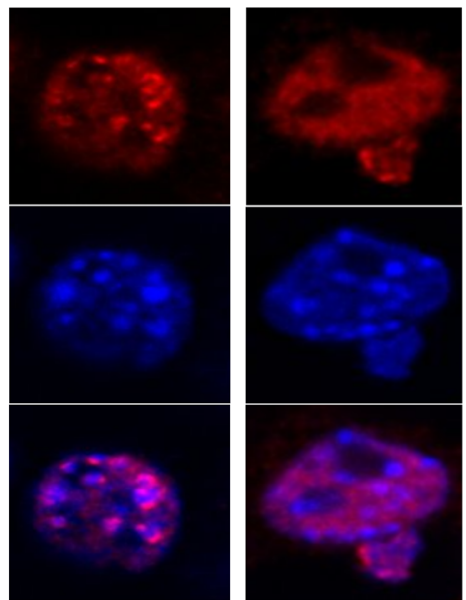

B

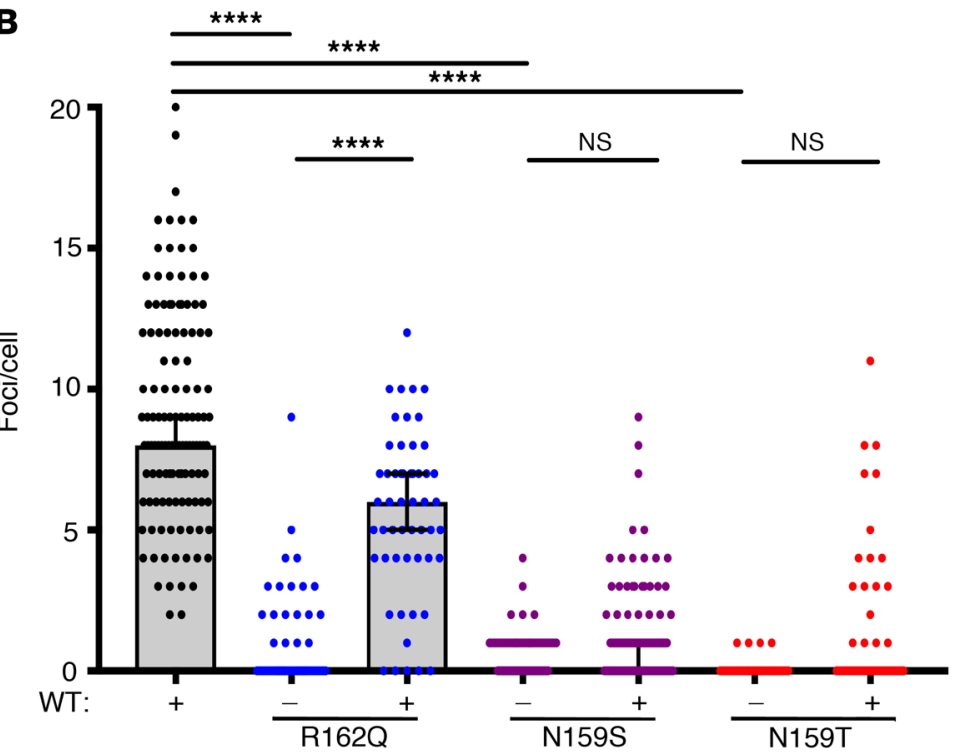

C

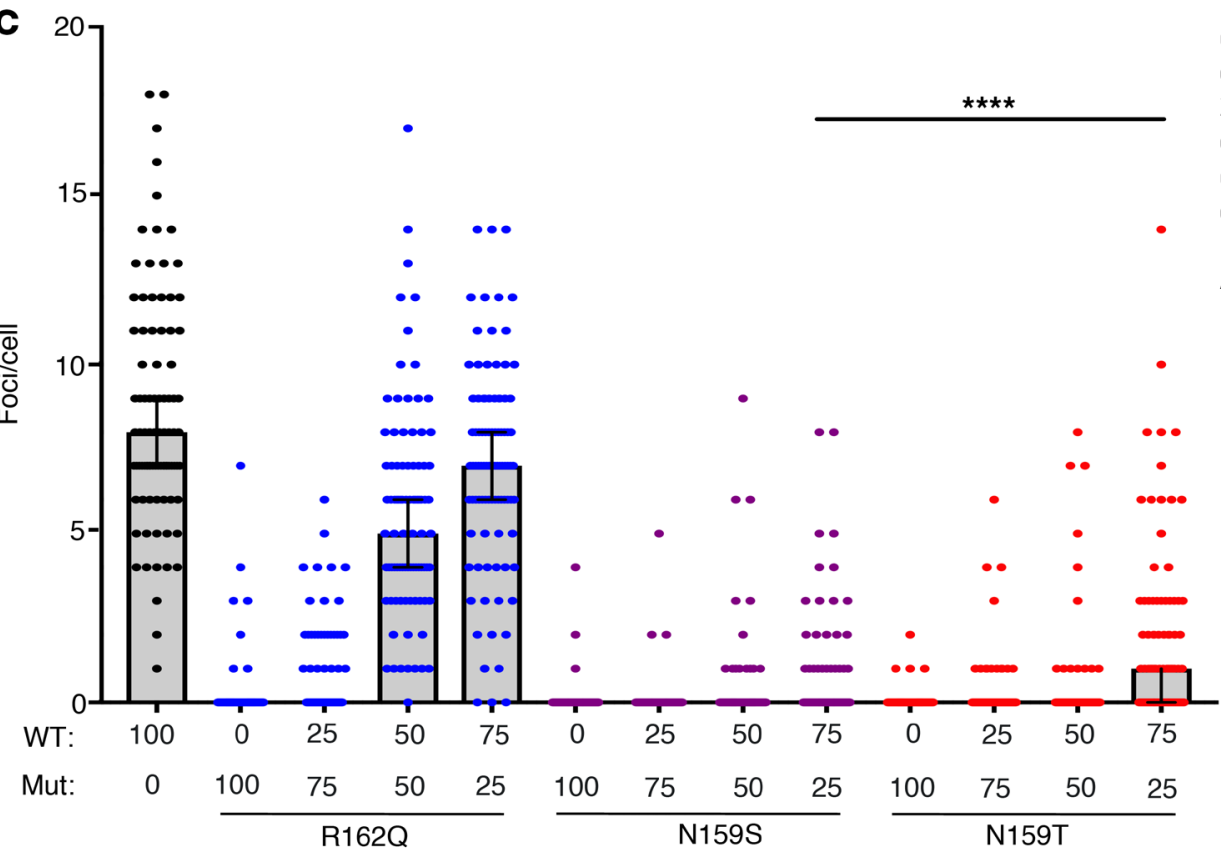

N159T

$+50 \%$ WT
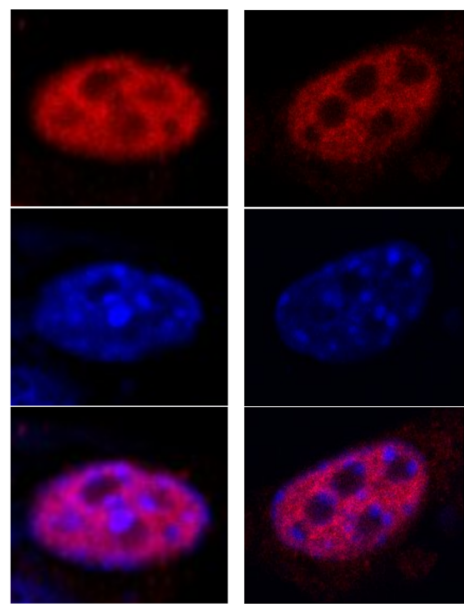

N159S

$+50 \%$ WT

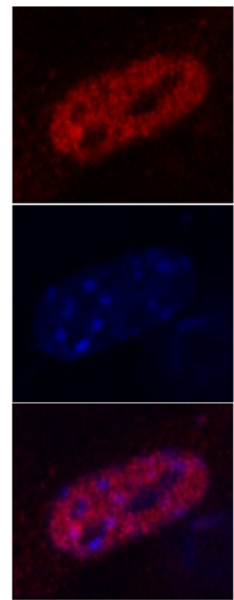

Figure 6. Interference of IKZF1 ${ }^{\text {N1595/T }}$ mutants with pericentromeric targeting of WT IKZF1. (A) NIH3T3 cells were transfected with WT IKZF1 or the indicated IKZF1 mutants (Mut) (R162Q, N159S, N159T) or with 1:1 WT/Mut ratio. After 36 hours, cells were labeled with a monoclonal anti-IKZF1 primary antibody and an Alexa Fluor 546-conjugated (red) secondary antibody plus DAPI. IKZF1 ${ }^{11229}$ haploinsufficient mutant was used for comparison. Cells were visualized by confocal microscopy, and images shown are representative of 5 experiments. Original magnification, $\times 190$. (B) Focus counts from the experiment in $\mathbf{A}$ IKZF1 foci were counted in 100 transfected NIH 3 T3 cells $(n=100)$ for each condition. Observer bias was eliminated by coding the slides prior to inspection. Statistical analysis was performed between the different groups with a 1-way ANOVA test. Horizontal lines represent the median $\pm 95 \%$ confidence interval (CI). ${ }^{* * *} P<0.0001$. (C) The experiment in $\mathbf{A}$ was repeated twice adding 3:1 and 1:3 WT/Mut ratio, and foci were enumerated as described in B. For each condition, 90-121 transfected cells were counted, and statistical analysis was performed between the different groups with a 1-way ANOVA test. Horizontal lines represent the median $\pm 95 \%$ Cl. ${ }^{* * *} P<0.0001$. 
We show that germline IKZF1 $1^{\mathrm{N} 159 \mathrm{~S} / \mathrm{T}}$ mutations behave in a dominant-negative fashion, contrasting with the germline and not-fully-penetrant haploinsufficient mutations previously described (18). A previous work by Hoshino et al. (19) did not show dominant-negative behavior of the IKZF1 ${ }^{\mathrm{N} 159 \mathrm{~S}}$ mutation, but this issue was not specifically addressed, as no enumeration of foci using different WT/mutant protein ratios was performed in their study. Moreover, the immunological and clinical phenotypes associated with IKZF1 $1^{\mathrm{N} 159 \mathrm{~S} / \mathrm{T}}$ mutations are broader, more severe, and consistently characterized by an early-onset CID in all patients studied. In addition, the fact that germline IKZF1 ${ }^{\mathrm{N} 159 \mathrm{~S} / \mathrm{T}}$ mutations were apparently de novo in all patients tested and no asymptomatic relatives or healthy individuals were detected suggests - although it cannot be formally assessed - a highly or even fully penetrant dominant disease (in contrast to that associated with haploinsufficient mutations). However, while $\mathrm{T}$ and $\mathrm{B}$ lymphoid defects were always present in IKZF1 $1^{\mathrm{N} 159 \mathrm{~S} / \mathrm{T}}$ patients, myeloid defects were variable, which suggests additional genetic and/or environmental factors. Interestingly, haploinsufficient mutations preserving one functionally active allele do not seem to impact $\mathrm{T}$ cell and myeloid biology in a clinically relevant manner $(15,18,22)$, which demonstrates that one fully functional IKZF1 copy is sufficient to retain normal $\mathrm{T}$ cell and myeloid functions.

Like haploinsufficient IKZF1 mutants, IKZF1 ${ }^{\mathrm{N1595} / \mathrm{T}}$ proteins failed to form foci with PC-HC localization and to bind $\gamma$-Sat8 and IK-bs4 DNA sequences, known targets for IKZF1. However, in contrast to their haploinsufficient counterparts, dominantnegative mutations inhibit formation of foci by WT IKZF1. Selected in silico modeling of IKZF1 3D structure predicted that serine and threonine substitutions of asparagine 159 may retain the ability to bind DNA but with altered specificity (Supplemental Figure 8 and data not shown). Therefore, some weak non-PC-HC DNA fixation of IKZF1 $1^{\mathrm{N} 159 \mathrm{~S} / \mathrm{T}}$ could help explain its dominant effect. Moreover, this potentially preserved DNA-binding ability could also modify the binding of WT IKZF1 and other partners, when heterodimerized with mutant IKZF1 $1^{\mathrm{N} 159 \mathrm{~S} / \mathrm{T}}$, as suggested by the absence of AIOLOS focus formation when coexpressed with IKZF1 ${ }^{\mathrm{N159S} / \mathrm{T}}$ (Supplemental Figure 7). More studies are needed to address the putative target sequences of IKZF1 ${ }^{\mathrm{N} 1595 / \mathrm{T}}$ dominant-negative mutants, ideally by use of a lymphoid system that expresses individual IKZF1 partners.

Taken together, these findings expand the clinical and biological spectrum associated with germline IKZF1 loss-of-function mutations in humans. These observations also provide insights into nonredundant functions of hematopoietic transcription factors, highlighting the key role of IKZF1 in hematopoiesis and T cell memory acquisition in humans.

\section{Methods}

Whole-exome sequencing and bioinformatics. See Supplemental Methods for whole-exome sequencing (WES) details for patients A1, C1, D1, E1, and G1. For patients B1 and F1, WES details have been previously provided elsewhere $(2,19)$. DNA sequencing exome data have been deposited into the NCBI's Sequence Read Archive (SRA SRP136278).

Sequence analysis and molecular modeling. The model of the 3D structure of the IKZF1 ZF1-4 DNA-binding domains was built with Modeller version 9.15 (38), on the basis of the alignment of its sequence with the PRMD9 ZNF8-11 (see Figure 1C), whose 3D structure has been solved in complex with a hot-spot oligonucleotide (20). Template selection was based on the results from Phyre2 (Structural Bioinformatics Group; fourth hit, confidence 99.7) and I-TASSER (Zhang Lab, https://zhanglab.ccmb.med.umich.edu/I-TASSER/; first hit, normalized $Z$ score 6.59), selecting for modeling arrays of fourth consecutive ZF, in complex with DNA. Molecular graphics and analyses were performed with the UCSF Chimera package (39). Sequence alignments were rendered using ENDscript (40).

Sanger sequencing. Selected NGS results were confirmed by Sanger sequencing. Specifically, IKZF1 coding exon 5 was amplified by PCR with exon-specific oligonucleotide primers and GoTaq Hot Start Polymerase (Promega) (forward primer sequence: CCCCCGTGGGAAACAACTTT; reverse primer sequence: GAGGGATCAGGGTTAGCCA). Purified PCR products were directly sequenced with BigDye Terminators (version 1.1, Thermo Fisher Scientific) and were analyzed on a 3130xL Genetic Analyzer (Applied Biosystems).

Plasmid constructs, cell transfections, and infections. Human IKAROS family zinc finger 1 (IKAROS) (IKZF1; NM_006060) cDNA was synthesized (GeneScript) and subcloned into the mammalian expression vector pcDNA3-HA or pCMV6-AC-DDK (Origene), pcDNA3.1D/V5-HisTOPO (Invitrogen). Indicated mutants for IKZF1 were generated based on the site-directed mutagenesis protocol with AccuPrime Pfx DNA Polymerase (Thermo Fisher Scientific) followed by DpnI treatment (Life Technologies) or Q5 Hot Start High Fidelity polymerase (Thermo Fisher Scientific), followed by KLD treatment (NEB). Human IKAROS family zinc finger 3 (IKZF3/AIOLOS) ORF clone (pcDNA3.1-AIOLOS, NM_012481) was purchased from GenScript.

Cell culture. PBMCs were isolated by the use of Ficoll separation (Lonza). PBMCs were cultured in RPMI 1640 with 10\% fetal bovine serum, $2 \mathrm{mM}$ L-glutamine, $100 \mathrm{U} / \mathrm{ml}$ penicillin, and $100 \mu \mathrm{g} / \mathrm{ml}$ streptomycin (Thermo Fisher Scientific) at $37^{\circ} \mathrm{C}$ in a humidified $5 \%$ $\mathrm{CO}_{2}$ incubator. NIH 3T3 and HEK293T (ATCC CRL-1658 and CRL11268) cells were cultured in DMEM with $10 \%$ fetal bovine serum, L-glutamine, and penicillin/streptomycin.

Expansion of $\mathrm{T}$ cell blasts was obtained by incubating PBMCs for 72 hours with PHA $(2.5 \mu \mathrm{g} / \mathrm{ml}$, Sigma-Aldrich) in Panserin 401 (PAN Biotech) supplemented with $5 \%$ human male Ab serum (BioWest), penicillin $(100 \mathrm{U} / \mathrm{ml})$, and streptomycin $(100 \mu \mathrm{g} / \mathrm{ml})$. After 3 days, dead cells were removed by Ficoll-Paque density gradient, and blasts were maintained in culture with IL-2 (100 UI/ml).

Fluorescence microscopy. NIH 3T3 cells $\left(0.8 \times 10^{5}\right.$ to $\left.1 \times 10^{5}\right)$ were seeded onto coverslips in 6-well plates. The next day, cells were transfected with indicated plasmid by Effectene (QIAGEN) or Lipofectamine 2000 (Thermo Fischer Scientific) according to the manufacturer's instructions. The cells were washed twice in PBS, fixed for 10 minutes in $4 \%$ paraformaldehyde, and permeabilized for 15 minutes in $0.1 \%$ Triton $\mathrm{X}-100$ in PBS at room temperature. The cells were then incubated for 30 minutes in blocking buffer (PBS with $10 \%$ FBS and $0.1 \%$ Triton $\mathrm{X}-100$ ) and then incubated for 2 hours with rabbit anti-IKZF1 monoclonal antibody (ab191394, Abcam, Figure 6A and Supplemental Figure 7A), anti-HA mouse monoclonal antibody (MMS-101P, Covance, Supplemental Figure 6 and Supplemental Figure 7C), anti-FLAG rabbit polyclonal affinity antibody (F7425, Sigma-Aldrich, Supplemental Figure 6), or anti-IKZF3 rabbit mAb (D1C1E, Cell Signaling Technology, Supplemental Figure 7). The cells were washed with PBS and incubated in blocking 
buffer for 1 hour with secondary antibodies conjugated with Alexa Fluor 546 (red color, Thermo Fisher Scientific) to reveal anti-IKZF1 staining, Alexa Fluor 488 (green color) to reveal anti-HA staining, or Alexa Fluor 568 (red color) to reveal anti-FLAG or anti-IKZF3 staining. Cells were washed and mounted on slides with VECTASHIELD Mounting Medium (Vector Laboratories). Images were collected by a ZOE fluorescent cell imager (Bio-Rad) or Leica SP8 STED confocal microscope (Leica Microsystems). For quantification of foci, foci per cell were manually counted under a confocal microscope on slides that were anonymized to the observer. About 100 cells were analyzed for each condition (see legend of Figure 6).

Lightshift chemiluminescent EMSA. HEK293T cells were transfected with PCMV-AC-DDK, WT or mutant, and nuclear extracts were prepared with NE-PER nuclear extract kit according to the manufacturer's instructions (Thermo Fisher Scientific). Gel mobility shift assays were performed with LightShift Chemiluminescent EMSA kit (Thermo Fisher Scientific) according to the manufacturer's instructions. DNAprotein complexes were separated on $6 \%$ Novex TBE gels with $0.5 \times \mathrm{TBE}$. Sequences for human probes were as follows: $\gamma$ satellite from human chromosome 8 ( $\gamma$-Sat8) (forward: 5'-BIOTIN-GCGAGACCGCAGGGAATGCTGGGAGCCTCCC; reverse: 5'-BIOTIN- GGGAGGCTCCCAGCATTCCCTGCGGTCTCGC) (41); IKAROS consensus-binding sequence 4 (IK-bs4) (forward: 5'-BIOTIN-TGACAGGGAATACACATTCCCAAAAGC; reverse: 5'-BIOTIN-GCTTTTGGGAATGTGTATTCCCTGTCA). EMSA bands were quantified by the Bio-Rad Image Lab program. Data were normalized with the binding of the extracts from cells transfected, with WT IKZF1 as 100\%.

Bone marrow staining. Bone marrow flow cytometric analysis was performed on a FACSCanto II flow cytometer (BD Biosciences), and immunohistochemistry was performed on bone marrow core biopsy sections by the Ventana automated staining system (Roche), as previously described (42). For the flow cytometric analysis, 500,000 total events were captured per bone marrow sample. Cells depicted are gated on all CD34 $4^{+}$cells and all CD19 ${ }^{+}$cells in bone marrow aspirate from a healthy control marrow (37,000 gated events) and patient B2 (14,000 gated events). The following antibodies (clone) were used: CD34 (8G12), CD19 (SJ25C1), TdT (E17-1519, BD Biosciences).

Cell proliferation. Human PBMCs were isolated by densitygradient centrifugation with Ficoll-Paque PLUS (GE Healthcare), washed twice in PBS, and cultured in complete RPMI-1640 medium containing $10 \% \mathrm{FBS}$ and penicillin-streptomycin-glutamine (Life Technologies) at $37^{\circ} \mathrm{C}$ in a humidified $5 \% \mathrm{CO}_{2}$ incubator. PBMCs, either freshly isolated or thawed from liquid nitrogen-stored samples, were incubated with CellTrace violet Cell Proliferation Kit (1 $\mu \mathrm{M}$; Invitrogen). After 20 minutes, 10 volumes of RPMI/10\% FBS were added, and the cells were centrifuged and washed twice more with RPMI/10\% FBS. A total of $1 \times 10^{5}$ cells were seeded into 96 -well plates, stimulated with coated anti-CD3 (precoated overnight at $4^{\circ} \mathrm{C}, 1$ or $10 \mu \mathrm{g} / \mathrm{ml}$, eBioscience), soluble anti-CD3 antibody and anti-CD28 antibody $(1 \mu \mathrm{g} / \mathrm{ml}$ each, clones $16-0037$ and 16-0288, eBioscience), anti-CD3/CD28-coated beads (Invitrogen), or PHA $(1 \mu \mathrm{g} / \mathrm{ml}$, Sigma-Aldrich) as indicated in the figures. IL-2 was used at a final concentration of $100 \mathrm{UI} / \mathrm{ml}$. Cells were stained with fluorochrome-conjugated CD4 and CD8 antibodies (BD Biosciences) for 30 minutes at $4^{\circ} \mathrm{C}$ (in the dark). Cells were washed with PBS twice, and cells were acquired and analyzed by flow cytometry (Becton Dickinson FACSCanto II) and FlowJo software (TreeStar).
Flow cytometry. For surface staining, PBMCs $\left(1 \times 10^{5}\right.$ to $5 \times 10^{5}$ cells per sample) were washed with PBS and then incubated for 30 minutes at $4^{\circ} \mathrm{C}$ (in the dark) with appropriate fluorochrome-labeled monoclonal antibodies or their isotype-matched control antibodies diluted in $100 \mu \mathrm{l} \mathrm{FACS} \mathrm{staining} \mathrm{buffer} \mathrm{(5 \%} \mathrm{FBS} \mathrm{in} \mathrm{PBS).} \mathrm{All} \mathrm{samples} \mathrm{were}$ acquired and analyzed by flow cytometry (Becton Dickinson FACSCanto II or LSRII-Fortessa) and FlowJo software.

The following validated antibodies were used for flow cytometry: anti-CD3 (SK7), anti-CD4 (RPA-T4), anti-CD8 (RPA-T8), anti-CD19 (SJ25C1), anti-CD20 (L27), anti-CD27 (LG.3A10), anti-CD62L (SK11), anti-CD122, anti-CD132, anti-CD197 (3D12), anti-TCR-V024 (C15) (BD Biosciences); anti-CD45RA (ALB11), anti-TCR-Vß11 (X21), antiTCR- $\gamma \delta$ (IMMU510), anti-IgM (G20-127), anti-IgD (IA6-2) (Beckman Coulter); anti-CD45RO (UCHL1) (Dako); anti-CD25 (BC96), anti-CD31 (WM59), anti-CD161 (HP-3G10), anti-TCR-Va7.2 (3C10) (Sony Biotechnology). For intracellular staining, PBMCs were fixed and permeabilized after surface staining with a FoxP3 staining kit (eBioscience), in accordance with the manufacturer's instructions, and then incubated with anti-Ikaros (clone R32-1149, BD Biosciences) for 30 minutes on ice. All samples were acquired and analyzed by flow cytometry (Becton Dickinson FACSCanto II) and FlowJo software.

DC phenotyping. DCs were defined as lineage negative (CD3-, $\mathrm{CD}^{-}, \mathrm{CD}^{-} 6^{-} \mathrm{CD}^{-}$, and $\mathrm{CD}^{-} 6^{-}$and HLA-DR positive populations. The CD303 versus CD11c plot enabled the delineation of plasmacytoid DCs (pDCs; CD11 $\mathrm{c}^{-} \mathrm{CD} 303^{+}$) and myeloid DCs (mDCs; $\left.\mathrm{CD} 11 \mathrm{c}^{+} \mathrm{CD} 303^{-}\right)$. The following validated antibodies were used: antiCD3 (OKT3), anti-CD14 (HCD14), anti-CD16 (3G8), anti-CD303 (201A) (BioLegend), anti-CD19 (1D3), anti-HLA-DR (G46-6) (BD Biosciences), anti-CD56 (HCD56), anti-CD11c (clone 3.9) (Sony Biotechnology).

Cytokine measurement. For intracellular staining of cytokines, day$7 \mathrm{~T}$ cell blasts were stimulated overnight with anti-CD3/CD28-coated beads (Invitrogen) or PMA $(20 \mathrm{ng} / \mathrm{ml})$ plus ionomycin $(1 \mu \mathrm{g} / \mathrm{ml})$ in the presence of brefeldin A (GolgiPlug, BD). Cells were fixed and permeabilized with the BD Cytofix/Cytoperm Plus kit (BD Pharmingen) according to manufacturer's instructions. Cells were then labeled with APC-anti-IFN- $\gamma$ (mouse IgG1, 4S.B3) or PE-anti-IL2 (rat IgG2a, MQ1-17H12) and isotype-matched monoclonal antibodies purchased from BioLegend. Cells were finally collected, washed, and stained with BV785-anti-CD3, BV510-anti-CD4, and/or BV650-PE-anti-CD8 mAbs and analyzed by flow cytometry.

For further identification of CD4 ${ }^{+}$Th1, Th2, and Th17 populations, total PBMCs were stimulated with PMA (100 ng/ml, Sigma-Aldrich) and ionomycin ( $1 \mu \mathrm{M}$, Sigma-Aldrich) for 6 hours in the presence of brefeldin A (10 $\mu \mathrm{g} / \mathrm{ml}$, Invitrogen). The stimulated cells were stained with PerCP-Cy5.5-anti-CD3 and PE-Cy7-anti-CD4 for an additional 30 minutes. Then cells were fixed, permeabilized with the BD Cytofix/ Cytoperm plus kit, incubated with the indicated antibodies (FITC-antiIL-2 [clone MQ1-17H12], PE-anti-IFN- $\gamma$ [clone B27], BV421-anti-IL-4 [clone 8D4-8], eFluor 660-IL-17A [clone eBio64CAP17, eBioscience]), and analyzed by flow cytometry.

For TCR- or TLR-induced cytokine generation, PBMCs $\left(1 \times 10^{6} /\right.$ $\mathrm{ml}$ ) were stimulated with Dynabeads T cell activator CD3/CD28 or various TLR agonists (Invivogen Human TLR1-9 agonist kit) for 24 hours, respectively. Cell-free supernatants were harvested, and cytokines were measured simultaneously with Fluorokine MAP Human Base Kit A (R\&D Systems) by the Luminex 200 System (Luminex Corp.). 
Immunoblotting. $\mathrm{T}$ cell blasts on day 14 of culture were lysed in sample buffer. Proteins were separated by SDS-PAGE and transferred on PVDF membranes (Millipore). Membranes were blocked with milk for 1 hour before incubation with primary antibodies. The following antibodies were used for immunoblotting: anti-IKZF1 (ab191394), purchased from Abcam, and anti-KU80 (2753S), purchased from Cell Signaling Technology. Membranes were then washed and incubated with anti-mouse or anti-rabbit HRP-conjugated antibodies from Cell Signaling and GE Healthcare, respectively. Pierce Enhanced Chemiluminescence Western blotting substrate was used for detection.

HEK293T cells were transfected with pcDNA3.1-IKZF1 WT or indicated mutant with the Lipofectamine 2000. Cells were then harvested for 36 hours, subsequently lysed in sample buffer, and immunoblotted with anti-IKZF1 and anti-KU80 antibodies.

Method for RNA sequencing and analysis. $\mathrm{CD}^{+} / \mathrm{CD}^{+} / \mathrm{CD}^{2} 5 \mathrm{RA}^{+}$ cells (naive $\mathrm{CD}^{+} \mathrm{T}$ cells) were enriched by negative selection with EasySep human naive $\mathrm{CD} 4^{+} \mathrm{T}$ cell isolation kit from patients' and controls' PBMCs; CD14 ${ }^{+}$cells (monocytes) were enriched by the EasySep human monocyte enrichment kit (STEMCELL Technologies) from patients' and controls' PBMCs and stimulated with LPS (100 ng/ml) for 24 hours. Purity was greater than $90 \%$ for both populations studied. Targeted RNA sequencing library preparation was carried out with an Ion AmpliSeq Transcriptome Human Gene Expression Kit (Life Technologies), which is designed to profile more than 20,000 distinct human reference sequence genes with a highly multiplexed amplification method. Each amplicon represents a unique targeted gene (one transcript per gene). The average size of each amplicon is approximately 150 bp. For library preparation, each sample was run in duplicate, and a cDNA library was first generated from a minimum of $10 \mathrm{ng}$ of total RNA. The cDNA was barcoded and amplified with Ion AmpliSeq technology, and the amplified cDNA Libraries were evaluated for quality and quantified with Agilent Bioanalyzer high-sensitivity chip. Libraries were then diluted to $100 \mathrm{pM}$ and pooled equally, with 4 individual samples per pool. Pooled libraries were amplified and enriched with the Ion Chef System (Life Technologies). Templated libraries were then sequenced on an Ion Torrent Proton sequencing system (Life Technologies) with Ion PI HiQ kit and chip version 3. We performed gene-level differential expression analysis of targeted RNA sequencing data on naive $\mathrm{CD} 4^{+}$cells and monocytes with the Bioconductor DESeq2 package (43). Briefly, for each cell type (naive CD4 ${ }^{+}$ and monocytes), we merged raw count files, systematically filtered out transcripts with low expression, and defined groups (control, patient), genotypes (IKZF1 WT, IKZF1 mutant), and phenotypes (healthy, haploinsufficient, dominant negative). The genotype and phenotype factors were combined to assess the interaction of these 2 factors. We then constructed a DESeq2 object, estimated size factors, $\log _{2}-$ normalized counts, collapsed the technical replicates, contrasted groups, annotated results, transformed the data (variance stabilization), and performed Pearson correlation.

RNA sequencing data have been deposited into the NCBI's Gene Expression Omnibus databases (GEO GSE112706 and GSE112707) and Sequence Read Archive database (SRA SRP136927 and SRP136939).
Statistics. Where indicated, data were by 1-way ANOVA test with GraphPad Prism software (GraphPad). The differences were considered significant when $P$ was less than 0.05 .

Study approval. All patients or their guardians provided written informed consent. The study and protocols conform to the $1975 \mathrm{Dec}-$ laration of Helsinki under institutional review boards, as well as to local legislation and ethical guidelines from the Comite de Protection des Personnes de l'Ile de France II and the French advisory committee. Blood from healthy donors and patients was obtained under approved protocols (see above) at the NIH, internal, and institutional review boards, which also allow for the collection and use of patients' family history and pedigrees for publication. All procedures were based on standard of care, and established clinical guidelines were followed.

\section{Author contributions}

DB, HSK, ZVW, and CL designed and performed experiments, and analyzed the data and clinical information. IC and JEN performed in silico analyses and modeling. SC and JWV performed TREC analysis. CP, AF, VB, CF, FV, NY, SK, HK, AH, FH, LL, VA, TF, RM, $\mathrm{KZ}$, JR, DA, JS, KRC, SH, SL, and SR identified the patients, and provided and analyzed clinical information. DB and HSK participated in the writing of the manuscript. SL and SR wrote the manuscript, interpreted data, and designed and supervised the research.

\section{Acknowledgments}

We acknowledge the patients, their families, and healthy donors for cooperation and blood donation. We thank the Plateforme UFR Necker for technical support. We also thank Wasantha Ranatunga and Roshini Abraham, Department of Laboratory Medicine and Pathology, Mayo Clinic, Rochester, Minnesota, USA, for their help with the CD4OL/CD40 studies; and Cyndi Tifft, Ellen Mcnamara, and Leslie Biesecker for their genetics advice. SL is a senior scientist at Centre National de la Recherche Scientifique-CNRS (France). DB is supported by the Agence Nationale de la Recherche (ANR). This work was supported by grants from Ligue Contre le Cancer-Equipe Labellisée (France) (to SL), Inserm (to SL), Agence Nationale de la Recherche (ANR-14-CE14-0028-01 to SL and ANR10-IAHU-01 to Institut Imagine). Exome sequencing was funded by the Rare Diseases Foundation (France) (to SL). This work was also supported by the Intramural Research Program, NIH Clinical Center. The content of this article does not necessarily reflect the views or policies of the Department of Health and Human Services, nor does mention of trade names, commercial products, or organizations imply endorsement by the US government.

Address correspondence to: Sylvain Latour, Laboratory of Lymphocyte Activation and Susceptibility to EBV Infection, Inserm UMR 1163, Institut Imagine, 24 Bd du Montparnasse, 75015 Paris, France. Phone: 33.1.42.75.43.03; Email: sylvain.latour@inserm.fr. Or to: Sergio D. Rosenzweig, Immunology Service, CC, DLM, NIH, 10 Center Dr., Bldg. 10, Rm. 2C410F Bethesda, Maryland 20892, USA. Phone: 301.451.8971; Email: srosenzweig@cc.nih.gov.
1. Molnár A, et al. The Ikaros gene encodes a family of lymphocyte-restricted zinc finger DNA binding proteins, highly conserved in human and mouse. J Immunol. 1996;156(2):585-592.
2. Yoshida N, et al. Germline IKAROS mutation associated with primary immunodeficiency that progressed to T-cell acute lymphoblastic leukemia. Leukemia. 2017;31(5):1221-1223.
3. John LB, Ward AC. The Ikaros gene family: transcriptional regulators of hematopoiesis and immunity. Mol Immunol. 2011;48(9-10):1272-1278.

4. Thomas RM, et al. Ikaros silences T-bet 
expression and interferon-gamma production during Thelper 2 differentiation. J Biol Chem. 2010;285(4):2545-2553.

5. Heizmann B, Sellars M, Macias-Garcia A, Chan S, Kastner P. Ikaros limits follicular B cell activation by regulating $\mathrm{B}$ cell receptor signaling pathways. Biochem Biophys Res Commun. 2016;470(3):714-720.

6. Dumortier A, Kirstetter P, Kastner P, Chan S. Ikaros regulates neutrophil differentiation. Blood. 2003;101(6):2219-2226.

7. Malinge S, et al. Ikaros inhibits megakaryopoiesis through functional interaction with GATA-1 and NOTCH signaling. Blood. 2013;121(13):2440-2451.

8. Rao KN, Smuda C, Gregory GD, Min B, Brown MA. Ikaros limits basophil development by suppressing C/EBP- $\alpha$ expression. Blood. 2013;122(15):2572-2581.

9. Keys JR, et al. A mechanism for Ikaros regulation of human globin gene switching. Br J Haematol. 2008;141(3):398-406.

10. Georgopoulos K, et al. The Ikaros gene is required for the development of all lymphoid lineages. Cell. 1994;79(1):143-156.

11. Kim J, et al. Ikaros DNA-binding proteins direct formation of chromatin remodeling complexes in lymphocytes. Immunity. 1999;10(3):345-355.

12. Georgopoulos K. Haematopoietic cell-fate decisions, chromatin regulation and ikaros. Nat Rev Immunol. 2002;2(3):162-174.

13. Wang JH, et al. Selective defects in the development of the fetal and adult lymphoid system in mice with an Ikaros null mutation. Immunity. 1996;5(6):537-549.

14. Papathanasiou P, et al. Widespread failure of hematolymphoid differentiation caused by a recessive niche-filling allele of the Ikaros transcription factor. Immunity. 2003;19(1):131-144.

15. Winandy S, Wu P, Georgopoulos K. A dominant mutation in the Ikaros gene leads to rapid development of leukemia and lymphoma. Cell. 1995;83(2):289-299.

16. Cobb BS, Morales-Alcelay S, Kleiger G, Brown KE, Fisher AG, Smale ST. Targeting of Ikaros to pericentromeric heterochromatin by direct DNA binding. Genes Dev. 2000;14(17):2146-2160.

17. Kuehn HS, et al. Loss of B cells in patients with heterozygous mutations in IKAROS. $\mathrm{N} \mathrm{EnglJ}$ Med. 2016;374(11):1032-1043.

18. Mullighan CG, et al. Deletion of IKZF1 and prognosis in acute lymphoblastic leukemia. $N$ Engl J Med. 2009;360(5):470-480.

19. Lana T, et al. Refinement of IKZF1 status in pedi- atric Philadelphia-positive acute lymphoblastic leukemia. Leukemia. 2015;29(10):2107-2110.

20. Hoshino A, et al. Abnormal hematopoiesis and autoimmunity in human subjects with germline IKZF1 mutations. JAllergy Clin Immunol. 2017;140(1):223-231.

21. Patel A, Hashimoto H, Zhang X, Cheng X. Characterization of How DNA Modifications Affect DNA Binding by $\mathrm{C} 2 \mathrm{H} 2 \mathrm{Zinc}$ Finger Proteins. Meth Enzymol. 2016;573:387-401.

22. Abdulhay N, et al. Normal hematologic parameters and fetal hemoglobin silencing with heterozygous IKZF1 mutations. Blood. 2016;128(16):2100-2103.

23. Duncan CJ, et al. Human IFNAR2 deficiency: Lessons for antiviral immunity. Sci Transl Med. 2015;7(307):307ra154.

24. Jouanguy E, Altare F, Lamhamedi-Cherradi S, Casanova JL. Infections in IFNGR-1deficient children. J Interferon Cytokine Res. 1997;17(10):583-587.

25. Dorman SE, Holland SM. Mutation in the signaltransducing chain of the interferon- $\gamma$ receptor and susceptibility to mycobacterial infection. J Clin Invest. 1998;101(11):2364-2369.

26. Adams SP, Kricke S, Ralph E, Gilmour N, Gilmour KC. A comparison of TRECs and flow cytometry for naive T cell quantification. Clin Exp Immunol. 2018;191(2):198-202.

27. Saunders AE, Johnson P. Modulation of immune cell signalling by the leukocyte common tyrosine phosphatase, CD45. Cell Signal. 2010;22(3):339-348.

28. Fischer A, Notarangelo LD, Neven B, Cavazzana M, Puck JM. Severe combined immunodeficiencies and related disorders. Nat Rev Dis Primers. 2015;1:15061.

29. Courtois G, et al. A hypermorphic IkappaBalpha mutation is associated with autosomal dominant anhidrotic ectodermal dysplasia and $\mathrm{T}$ cell immunodeficiency. J Clin Invest. 2003;112(7):1108-1115.

30. Pérez de Diego R, et al. Genetic errors of the human caspase recruitment domain-B-cell lymphoma 10-mucosa-associated lymphoid tissue lymphoma-translocation gene 1 (CBM) complex: Molecular, immunologic, and clinical heterogeneity. J Allergy Clin Immunol. 2015;136(5):1139-1149.

31. Schober T, et al. A human immunodeficiency syndrome caused by mutations in CARMIL2. Nat Commun. 2017;8:14209.
32. Trung NT, Kremmer E, Mittler G. Biochemical and cellular characterization of transcription factors binding to the hyperconserved core promoter-associated M4 motif. BMC Genomics. 2016;17:693.

33. de la Morena MT, et al. Long-term outcomes of 176 patients with X-linked hyper-IgM syndrome treated with or without hematopoietic cell transplantation. J Allergy Clin Immunol. 2017;139(4):1282-1292.

34. Hanna S, Etzioni A. MHC class I and II deficiencies. JAllergy Clin Immunol. 2014;134(2):269-275.

35. Kotlarz D, et al. Loss-of-function mutations in the IL-21 receptor gene cause a primary immunodeficiency syndrome. JExp Med. 2013;210(3):433-443.

36. Spinner MA, et al. GATA2 deficiency: a protean disorder of hematopoiesis, lymphatics, and immunity. Blood. 2014;123(6):809-821.

37. Olsson L, Johansson B. Ikaros and leukaemia. Br J Haematol. 2015;169(4):479-491.

38. Martí-Renom MA, Stuart AC, Fiser A, Sánchez $\mathrm{R}$, Melo F, Sali A. Comparative protein structure modeling of genes and genomes. Annu Rev Biophys Biomol Struct. 2000;29:291-325.

39. Pettersen EF, et al. UCSF Chimera - a visualization system for exploratory research and analysis. JComput Chem. 2004;25(13):1605-1612.

40. Robert X, Gouet P. Deciphering key features in protein structures with the new ENDscript server. Nucleic Acids Res. 2014;42(Web Server issue):W320-W324.

41. Ronni T, Payne KJ, Ho S, Bradley MN, Dorsam $G$, Dovat S. Human Ikaros function in activated $\mathrm{T}$ cells is regulated by coordinated expression of its largest isoforms. J Biol Chem. 2007;282(4):2538-2547.

42. Dulau Florea AE, et al. Abnormal B-cell maturation in the bone marrow of patients with germline mutations in PIK3CD. JAllergy Clin Immunol. 2017;139(3):1032-1035.e6.

43. Love MI, Huber W, Anders S. Moderated estimation of fold change and dispersion for RNA-seq data with DESeq2. Genome Biol. 2014;15(12):550.

44. Shearer WT, et al. Lymphocyte subsets in healthy children from birth through 18 years of age: the Pediatric AIDS Clinical Trials Group P1009 study. JAllergy Clin Immunol. 2003;112(5):973-980.

45. Schatorjé EJ1, Gemen EF, Driessen GJ, Leuvenink J, van Hout RW, de Vries E. Paediatric reference values for the peripheral $\mathrm{T}$ cell compartment. Scand J Immunol. 2012;75(4):436-444. 